ARTICLE

DOI: $10.1038 / s 41467-018-06648-6$

\title{
Incompatibility of the circadian protein BMAL1 and HNF $4 \alpha$ in hepatocellular carcinoma
}

Baharan Fekry ${ }^{1}$, Aleix Ribas-Latre ${ }^{1}$, Corrine Baumgartner ${ }^{1}$, Jonathan R. Deans ${ }^{2}$, Christopher Kwok ${ }^{1}$, Pooja Patel ${ }^{3}$, Loning $\mathrm{Fu}^{3}$, Rebecca Berdeaux ${ }^{1,4}$, Kai Sun ${ }^{1,5}$, Mikhail G. Kolonin (1) , Sidney H. Wang ${ }^{1}$, Seung-Hee Yoo ${ }^{5}$, Frances M. Sladek ${ }^{2}$ \& Kristin Eckel-Mahan (1) 1,5

Hepatocyte nuclear factor 4 alpha $(H N F 4 \alpha)$ is a master regulator of liver-specific gene expression with potent tumor suppressor activity, yet many liver tumors express HNF4 $\alpha$. This study reveals that $\mathrm{P} 1-\mathrm{HNF} 4 \alpha$, the predominant isoform expressed in the adult liver, inhibits expression of tumor promoting genes in a circadian manner. In contrast, an additional isoform of HNF4 $\alpha$, driven by an alternative promoter (P2-HNF4 $\alpha$ ), is induced in HNF4 $\alpha$-positive human hepatocellular carcinoma (HCC). P2-HNF4 $\alpha$ represses the circadian clock gene ARNTL (BMAL1), which is robustly expressed in healthy hepatocytes, and causes nuclear to cytoplasmic re-localization of $\mathrm{P} 1-\mathrm{HNF} 4 \alpha$. We reveal mechanisms underlying the incompatibility of BMAL1 and P2-HNF4 $\alpha$ in HCC, and demonstrate that forced expression of BMAL1 in HNF4 $\alpha$-positive HCC prevents the growth of tumors in vivo. These data suggest that manipulation of the circadian clock in HNF4 $\alpha$-positive HCC could be a tractable strategy to inhibit tumor growth and progression in the liver.

\footnotetext{
${ }^{1}$ Institute of Molecular Medicine, McGovern Medical School at the University of Texas Health Science Center (UT Health), Houston, TX 77030, USA. ${ }^{2}$ Department of Molecular, Cell and Systems Biology, University of California Riverside, Riverside, CA 92521, USA. ${ }^{3}$ Department of Pediatrics, Molecular and Cellular Biology, Children's Nutrition Research Center, Baylor College of Medicine, Houston, TX 77030, USA. ${ }^{4}$ Department of Integrative Biology and Pharmacology, McGovern Medical School at the University of Texas Health Science Center (UT Health), Houston, TX 77030, USA. ${ }^{5}$ Department of Biochemistry and Molecular Biology, McGovern Medical School at the University of Texas Health Science Center (UT Health), Houston, TX 77030, USA. Correspondence and requests for materials should be addressed to K.E-M. (email: Kristin.L.Mahan@uth.tmc.edu)
} 
epatocellular carcinoma (HCC) is the leading hepatic malignancy found in humans and the second leading cause of all malignancy-related cancer deaths ${ }^{1}$. HCC is on the rise in the US and elsewhere, and has been linked to the increased incidence of nonalcoholic fatty liver disease, which is driven by the obesity epidemic ${ }^{2}$. Unfortunately, tumors are often found at a late stage with limited potential for surgical removal, making efforts to elucidate the mechanisms responsible for HCC tumor growth and metastasis paramount for improving patient prognosis.

The circadian clock is an intrinsic, 24-h time keeping system that operates in all cells of the body, governing rhythmicity in cell function including metabolism, gene expression, and trafficking and transport of cellular proteins ${ }^{3-6}$. Circadian disruption in humans has been linked to a number of diseases, including cancer $^{7-16}$. Furthermore, experiments that mimic human jet-lag in mice reveal that circadian disruption is sufficient to induce spontaneous $\mathrm{HCC}^{17}$. The transcriptional activators, circadian locomoter output cycles kaput protein (CLOCK) and aryl hydrocarbon receptor nuclear translocator like (ARNTL, also known as BMAL1) form a heterodimer in hepatocytes and other cell types, and are necessary to drive the circadian transcription necessary for rhythmicity in many cellular events ${ }^{6,18}$.

Hepatocyte nuclear factor $4 \alpha$ (HNF4a) was originally identified as a nuclear factor enriched in the liver and important for control of genes involved in hepatocyte fate determination and function 19,20 . Since then, diverse roles for HNF4a have been described ${ }^{16,21-26}$, including its ability to function as a tumor suppressor, suppressing several genes (such as cyclin D1, CCND1) known to be causal for HCC initiation, growth, or progression ${ }^{27-35}$. While generally considered a transcriptional activator, several repressor proteins can also interact with HNF4a $\alpha^{22,36,37}$, allowing repression of some target genes. Some evidence suggests that human HCC may be deficient in HNF4a expression ${ }^{32}$, and overexpression of one of its isoforms in mice reverses the growth of liver tumors arising from chemically induced mutations ${ }^{32}$, while depletion of this isoform promotes tumor growth ${ }^{38}$.

Complicating the mechanistic understanding of HNF4a in liver cancer is the fact that there are two promoters (P1 and P2) that drive the expression of different $H N F 4 a$ transcript variants, which are differentially expressed not just in human HCC, but also colon cancer $28,39,40$. The P1 promoter gives rise to HNF4a1/2 which is expressed in normal adult liver, while the P2 promoter gives rise to $\mathrm{HNF} 4 a 7 / 8$, which is not normally expressed in the adult liver, but is in fetal liver as well as $\mathrm{HCC}^{39,41}$. While P1HNF4a is typically found only in the nucleus, posttranslational modifications can promote cytoplasmic trafficking $40,42,43$.

Our results reveal that the two isoforms of HNF4a ("P1HNF4 $\alpha$ " and "P2-HNF4a"), which are differentially expressed in liver cancer, exhibit distinct circadian roles. While P1-HNF4a normally represses cell cycle and epithelial-to-mesenchymal transition (EMT) genes in a circadian manner, P2-HNF4a is selectively induced in HCC, where it directly inhibits the expression of the circadian protein BMAL1 and leads to the cytoplasmic expression of the P1 isoform. Importantly, forced expression of BMAL1 in HNF4a-positive liver cancer cells impairs spheroid growth in culture and tumor growth in vivo, demonstrating that manipulation of the circadian clock in HNF4a-positive HCC could be a realistic strategy to slow or reverse growth of human HCC.

\section{Results}

HNF4a is heterogeneously expressed in human HCC. While evidence suggests that HNF4a has tumor suppressive effects in the liver ${ }^{38}$, heterogeneity of HNF4a expression in HCC has largely been observed using antibodies that do not distinguish between the P1 and P2 isoforms. To reassess HNF4a heterogeneity in liver cancer, mouse and patient-derived human HCC and hepatoblastoma cell lines were first stained using an antibody recognizing both isoforms (P1 and P2) of HNF4a (Fig. 1a). Several HCC cell lines expressed P1/P2-HNF4a robustly while Hepa-1c1c7 cells lacked HNF4a. The nontransformed hepatocyte-derived AML12 cell line also expressed P1/P2HNF4a, as did the human cancer line HepG2, which is commonly used as an in vitro model for HCC, but is more appropriately classified as hepatoblastoma ${ }^{44,45}$ (Fig. 1a). Using PCR primers and immunoblotting reagents that recognize both the $\mathrm{P} 1$ and $\mathrm{P} 2$ isoforms, similar patterns were observed: Hepa-1c1c7 cells were devoid of P1/P2 transcripts and proteins, while AML12, HepG2, Huh7 and Hep3B cells all expressed HNF4a mRNA and protein (Fig. 1b, c). Because cells grown in two-dimensional (2D) culture do not always retain normal patterns of gene expression (reviewed in ${ }^{46}$ ), we cultured HNF4a-positive HepG2 cells and HNF4a-negative Hepa-1c1c7 cells in Matrigel to generate small 3D spheroids. HepG2 spheroids stained with an antibody recognizing both isoforms of $\mathrm{HNF} 4 a$ showed robust HNF4a expression while Hepa-1c1c7-derived spheroids were devoid of the protein (Fig. 1d). These results indicate that $2 \mathrm{D}$ vs. $3 \mathrm{D}$ growth conditions alone did not account for the presence or lack of HNF4a.

To expand our understanding of HNF4a heterogeneity in HCC, spontaneous HCC isolated from mice subjected to a protocol that simulates jet-lag in humans (so called "jet-lagged" mice) ${ }^{17}$ were stained for P1/P2-HNF4a protein. Heterogeneity in HNF4a expression was also observed in HCC specimens from jetlagged mice (Fig. 1e). Furthermore, analysis of microarray data from human HCC (R2: Genomics Analysis and Visualization Platform, http://hgserver1.amc.nl/cgi-bin/r2/main.cgi) revealed heterogeneity in HNF4a transcript, with HCC displaying extremely high or extremely low levels of $H N F 4 a$ mRNA compared to control tissue (Fig. 1f). Staining for P1/P2-HNF4a protein in human HCC arrays revealed that approximately half of HCC tumors are positive for P1/P2-HNF4a (Fig. $1 \mathrm{~g}$ and Supplementary Fig. 1a). While HCC is more common in males than females ${ }^{47}$, the heterogeneity in HNF4a expression was not sex-specific. HNF4a-positive tumors analyzed for intensity across grades, revealed that metastatic regions expressed higher levels of P1/P2-HNF4a compared to grades 1-3 or to cirrhotic and hyperplastic regions (Fig. 1g and Supplementary Fig. 1b). Thus, while nontransformed liver cells and control tissue express moderate levels of HNF4a, HCC tumors are heterogeneous in their expression of HNF4a, and HNF4a-positive tumors have significantly higher expression of the protein compared to normal liver (Fig. $1 \mathrm{~g}$ and Supplementary Fig. 1b).

HNF4a has circadian activity in liver cells. Based on studies suggesting that HNF4a may influence the liver circadian clock ${ }^{23}$, we examined whether a circadian function of HNF4a might differ in the context of HNF4a-positive HCC by evaluating target gene expression. Primary mouse hepatocytes and AML12 cells were serum shocked to synchronize the circadian clock across the cells. The expected circadian nuclear translocation of BMAL1 was observed in primary mouse hepatocytes following serum shock (Supplementary Fig. 1c) and Dbp (a canonical CLOCK:BMAL1 target gene) showed the expected circadian oscillation (Supplementary Fig. 1d, $P=0.0199$ ). To assess whether HCC and hepatoblastoma lines were able to synchronize in culture, Hepa1c1c7, Hep3B, and HepG2 cells were serum shocked and Dbp expression was examined. The cancer cells were also able to synchronize and sustain 24-h rhythmicity, as indicated 


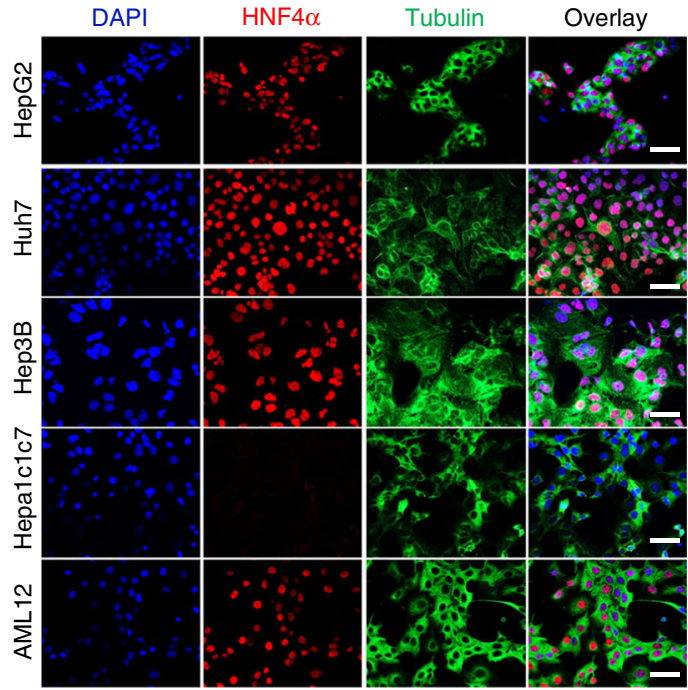

b
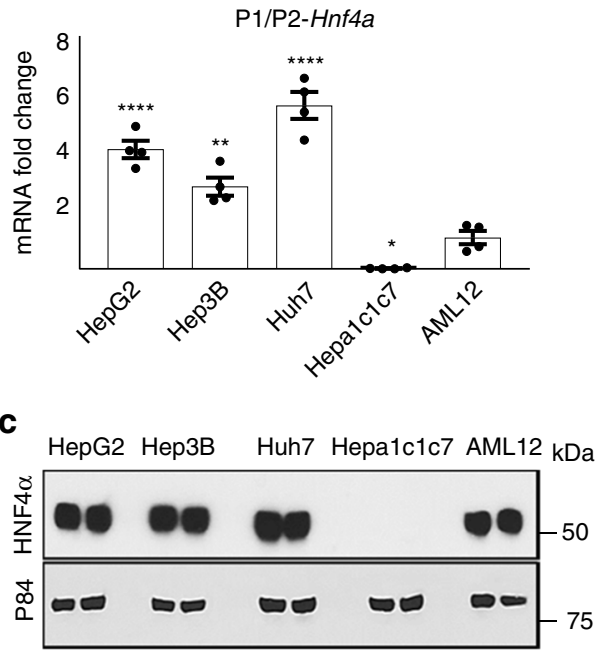

d

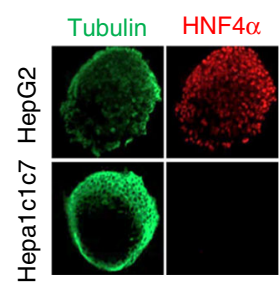

e

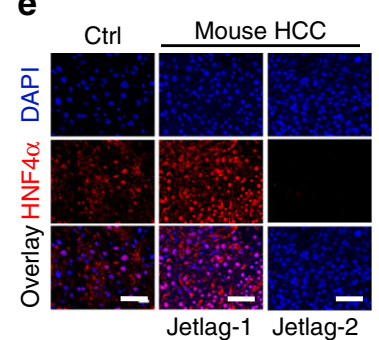

f

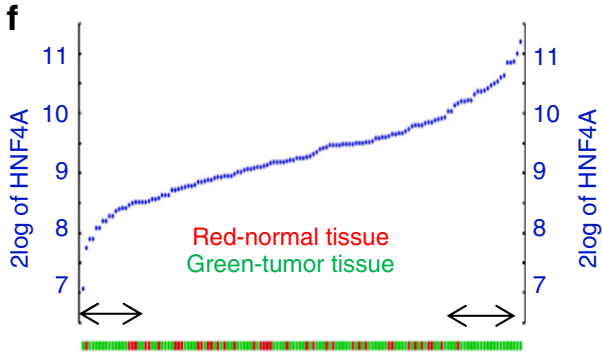

g

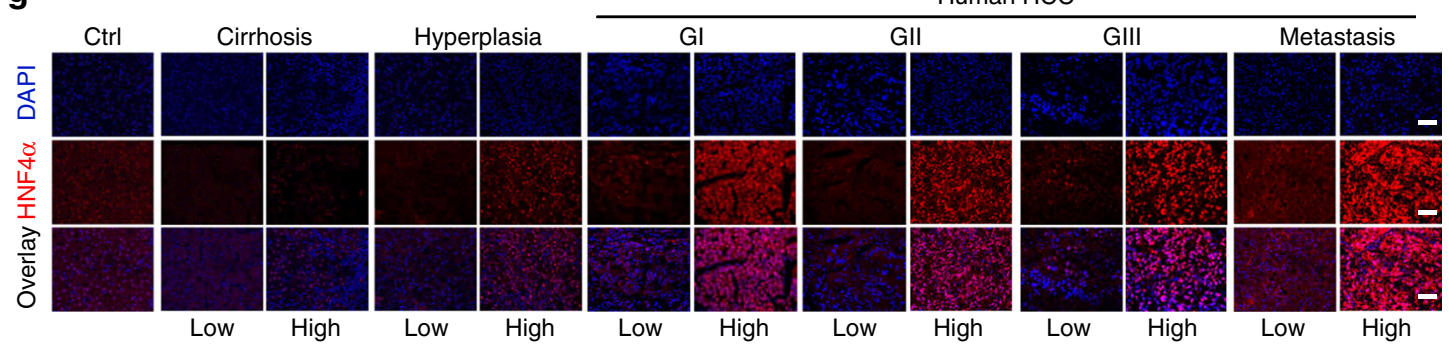

h $\rightarrow$ Scrambled $\rightarrow$ P1/P2-HNF4 $\alpha$ siRNA
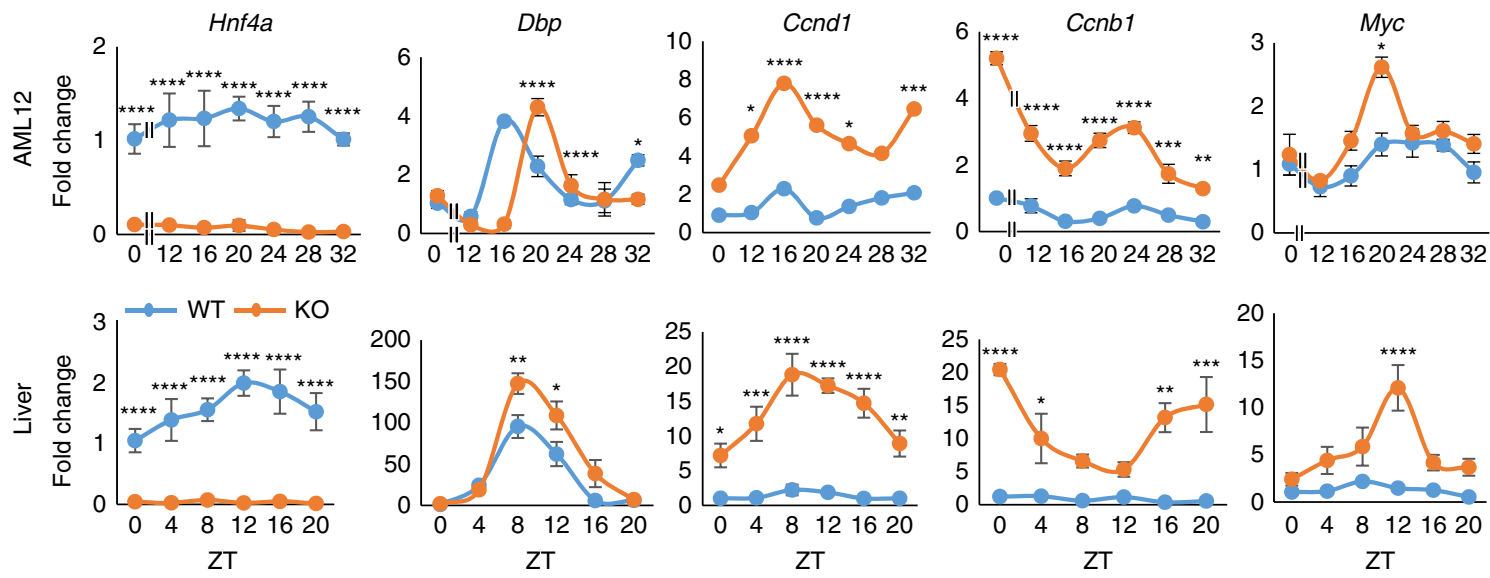

by oscillatory $D b p$ expression in Hepa-1c1c and Hep3B cells $(P=$ 0.027 and 0.004 , respectively, JTK_Cycle test for rhythmicity ${ }^{48}$, Supplementary Fig. 1d and Supplementary Table 1). To determine whether loss of HNF4a altered the circadian expression of target genes, we treated AML12 cells with siRNA against both P1/
P2 isoforms of Hnf4a. In addition, we inducibly knocked out HNF4a in the adult mouse liver using a previously described mouse model ${ }^{27}$. Reducing P1/P2-HNF4a expression in AML12 cells and healthy liver did not eliminate rhythmicity of the core clock genes, though Dbp was phase shifted in AML12 cells 
Fig. 1 HNF $4 \alpha$ is heterogeneously expressed in HCC. a Immunofluorescence (IF) reveals P1/P2-HNF4 $\alpha$ expression and subcellular localization in mouse and human HCC, hepatoblastoma cancer lines, and in the nontransformed liver cell line, AML12, grown in monolayer conditions. b RT-PCR reveals mRNA abundance of P1/P2-HNF4 $\alpha$ in HCC cell lines in vitro, fold change over AML12 Hnf4a mRNA. Compared to ZTO at the same time, ${ }^{\star} P<0.01,{ }^{\star \star} P<0.01,{ }^{\star \star \star} P$ $<0.001,{ }^{\star \star \star \star} P<0.0001$, one-way ANOVA test, Dunnett's multiple comparisons test. $(N=4)$. c Western blot showing P1/P2-HNF4 $\alpha$ abundance in whole cell lysates of cancer cell lines and nontransformed AML12 cells. d Staining of 3D, HCC- and hepatoblastoma-derived spheroids from HepG2 and Hepa1c1c7 cells with antibodies against P1/P2-HNF4 $\alpha$. e IF staining of spontaneous HCC isolated from jet-lagged WT mice using antibody to P1/P2-HNF4 $\alpha$. $\mathbf{f}$ mRNA expression of HNF4a in human HCC tumors and surrounding normal liver tissue (R2: genomics analysis and visualization platform). $\mathbf{g}$ IF staining of human normal ("Ctrl"), cirrhotic, hyperplastic, and HCC tumor tissue using antibody against P1/P2-HNF4 $\alpha$ ( $=$ grades, advancement increasing with number). h RT-PCR reveals the circadian expression of P1/P2-Hnf4a, Dbp, Ccnd1, Ccnb1 and Myc following the application of scrambled (Sc) or siRNA specific to P1/P2-Hnf4a in AML12 cells (top panel) or in the liver of WT and HNF4 $\alpha$ knockout (KO) mice (bottom panel). Compared to controls at the same time, ${ }^{\star} P<0.03,{ }^{\star \star} P<0.005,{ }^{\star \star \star} P<0.001,{ }^{\star \star \star \star} P<0.0001$, two-way ANOVA test, Sidak's multiple comparisons test. $(N=8-43)$. Scale bar is $50 \mu$ m. (See Supplementary Table 1 for JTK_Cycle Rhythmicity Statistics.) Error bars = SEM

(Fig. 1h, Supplementary Fig. 1e, f, and Supplementary Table 1). In contrast, $M y c$ expression was significantly increased in AML12 cells in the absence of P1/P2-HNF4a, and the HNF4a target genes, cyclin B1 (Ccnb1) and cyclin D1 (Ccnd1), which P1HNF4a has been shown to repress at single time points ${ }^{27}$, showed robust rhythmicity in expression in the HNF4a KO liver $(P=$ 0.0026 and $P=0.0075$, respectively) (Fig. $1 \mathrm{~h}$ and Supplementary Table 1), and an increase at the protein level (Supplementary Fig. 1g, h). Though the mechanisms are not known, HNF4a transcriptionally inhibited target genes at different phases. Being one of the most abundant hepatic transcription factors, HNF4a binds to over 20,000 locations in the genome ${ }^{49}$, and plausibly may activate or repress at different zeitgeber times based on the temporal array of different cofactors shared. Loss of P1/P2-HNF4a had no significant impact on the circadian expression of regulators of EMT, such as Snail (Snai1), Slug (Snai2), and Ecadherin (Cdh1), in either AML12 cells or the liver (Supplementary Fig. 1e, f and see Supplementary Table 1). To determine whether circadian de-repression of HNF4a target genes was BMAL1-dependent, we used siRNA to knockdown BMAL1 in the presence or absence of siRNA to P1/P2-Hnf4a and analyzed Ccnd1 expression following serum shock in AML12 cells (Supplementary Fig.1i). Interestingly, while loss of BMAL1 or P1/P2HNF4a both increased Ccnd1 expression, co-treatment did not further augment the effect (Supplementary Fig. 1j). Thus, nontransformed cells as well as HNF4a-positive and -negative HCC cells can exhibit 24-h rhythmicity, and in nontransformed liver cells, P1/P2-HNF4a represses the circadian expression of $M y c$, Ccnb1, and Ccnd1, which are involved in cell growth and proliferation.

Loss of HNF4a and BMAL1 co-expression in HCC. While BMAL1 and HNF4 $\alpha$ are co-expressed in healthy hepatocytes and nontransformed hepatocyte-derived cell lines (Fig. 2a-c, Supplementary Fig. 1a, g, h), we found that cancer cells expressing P1/ P2-HNF4a express little to no BMAL1. On the other hand, HCC that is devoid of HNF4a expresses high levels of BMAL1 mRNA and protein (Fig. 2a-c). Analysis of human HCC microarray data sets (R2: Genomics Analysis and Visualization Platform), revealed that ARNTL (BMAL1) and HNF4A are also inversely correlated at the level of mRNA expression in human HCC $(P<$ $0.0017 ; N=134$ ) (Fig. 2d). Both $2 \mathrm{D}$ and $3 \mathrm{D}$ culture of HepG2 cells confirmed that while the HNF4a-positive HepG2-cells did not express BMAL1 (Supplementary Fig. 2a), HNF4a-deficient Hepa-1c1c7 spheroids robustly expressed Bmall (Fig. 2b, $c$ and Supplementary Fig. 2b). HepG2 spheroids infected with a lentiviral construct for BMAL1 showed reduced HNF4a expression (Supplementary Fig. 2a), while Hepa-1clc7 spheroids infected with an HNF4a-containing vector showed reduced BMAL1 expression in synchronized organoid cultures (Supplementary
Fig. 2b), suggesting an inverse relationship between BMAL1 and HNF4a in liver cancer cells. Similarly, GFP-sorted HepG2 cells expressing GFP-BMAL1 showed reduced HNF4a, while GFPsorted Hepa-1c1c7 cells expressing GFP-HNF4a showed reduced Bmall mRNA abundance, confirming an inverse correlation between HNF4a and BMAL1 in the context of HCC (Supplementary Fig. 2c, d). To determine whether this apparent incompatibility of expression was present in spontaneous HCC, HCC from jet-lagged mice were stained with antibodies for BMAL1 and P1/P2-HNF4a. Spontaneous HCC from jet-lagged mice also revealed that high HNF4a expression coincided with low BMAL1 expression and vice versa (Fig. 2f). Finally, human HCC sections stained with antibodies to P1/P2-HNF4a and BMAL1 revealed the same inverse relationship (Fig. $2 \mathrm{~g}$ and Supplementary Fig. 2e).

P2-HNF4a is induced in HCC and has unique circadian activity. While the $\mathrm{P} 1$ promoter-driven isoform is mainly expressed in the liver of adult mice, both P1-HNF4a and P2$\mathrm{HNF} 4 \alpha$ are expressed in the normal gut ${ }^{39,40,50}$ (Supplementary Fig. 2F), where only the P1-HNF4a has tumor suppressive activity and aberrant expression of $\mathrm{P} 2-\mathrm{HNF} 4 a$ contributes to colitis-associated colon cancer $^{28,30}$. P2-HNF4a is also found in $\mathrm{HCC}^{39}$, and has recently been associated with poor prognosis ${ }^{51}$. Using primers detecting only P1-HNF4a or P2-HNF4a we analyzed the expression of each isoform across normal and transformed liver cells. While Hepa-1c1c7 had no detectable P1- or P2-HNF4a, SNU449, HepG2, Hep3B, and Huh7 cells expressed both P1- and P2-HNF4a or only P2-HNF4 (SNU449). P2HNF4a was not detectable in normal liver tissue (Fig. 3a). Antibodies specific to either P1-HNF4a or P2-HNF4a revealed that AML12 cells also do not express detectable P2-HNF4a, while all the HNF4a-positive cancer lines do (Fig. 3b, c). To determine whether P1-HNF4a is indeed responsible for the circadian transcriptional repression observed in Fig. 1h, HNF4a isoformspecific siRNA was administered to HepG2 cells. In control cells, robust oscillation of $P 2-H N F 4$ a was observed $(P=0.0008)$, compared to P1-HNF4a, which did not oscillate (Fig. 3d and Supplementary Table 1). Loss of both P1- and P2-HNF4a resulted in a pronounced upregulation of BMAL1, CCND1, and CCNB1 proteins and an upregulation of the BMAL1 target $D B P$, as well as circadian induction of CCND1 and CCNB1 transcripts after serum synchronization (Supplementary Fig. 3a, b). Interestingly, knockdown of only the P1 isoform of HNF4a did not affect BMAL1 abundance, while CCND1 and CCNB1 were greatly induced and CCND1 exhibited rhythmicity at the level of mRNA $\left(P=6.80 \mathrm{E}^{-05}\right)$ (Fig. 3e, $\left.\mathrm{f}\right)$. Overexpression of the $\mathrm{P} 1$ isoform in HNF4a-deficient Hepa-1c1c7 cells resulted in the inverse outcome, with the levels of CCND1 and CCNB1 being reduced at the mRNA and protein levels (Supplementary Fig. 3E). 
a
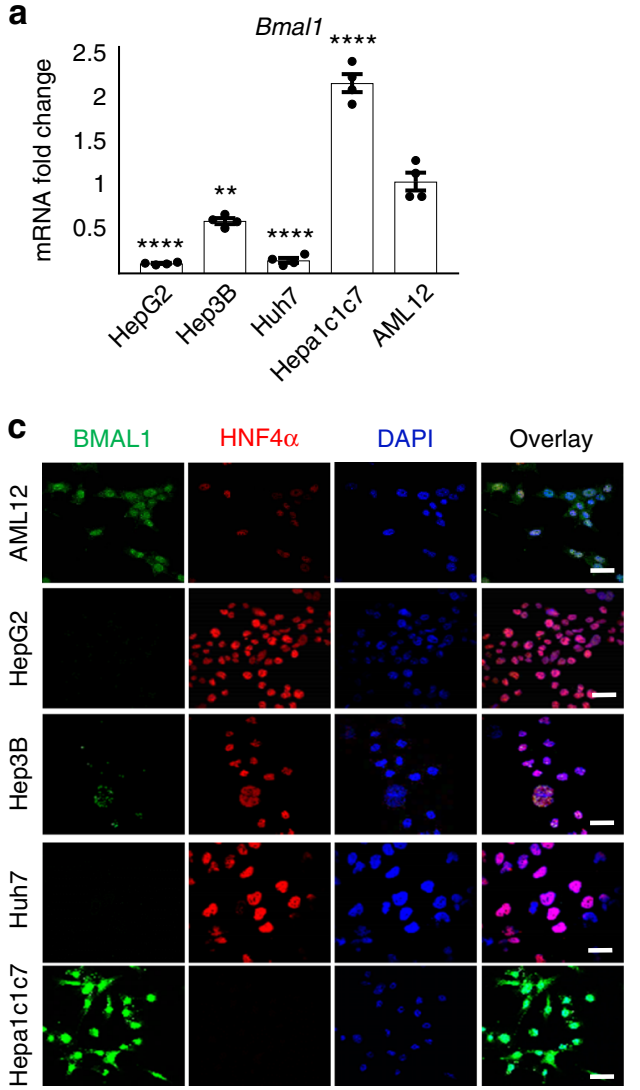

e HepG2

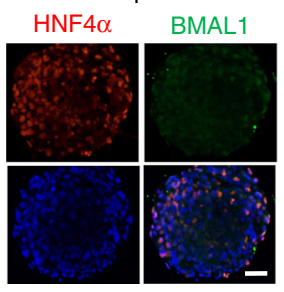

DAPI
Hepa1c1c7

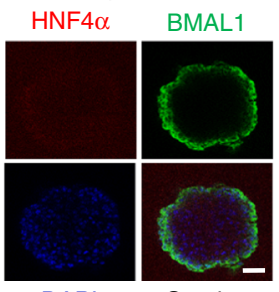

DAPI

Overlay

g

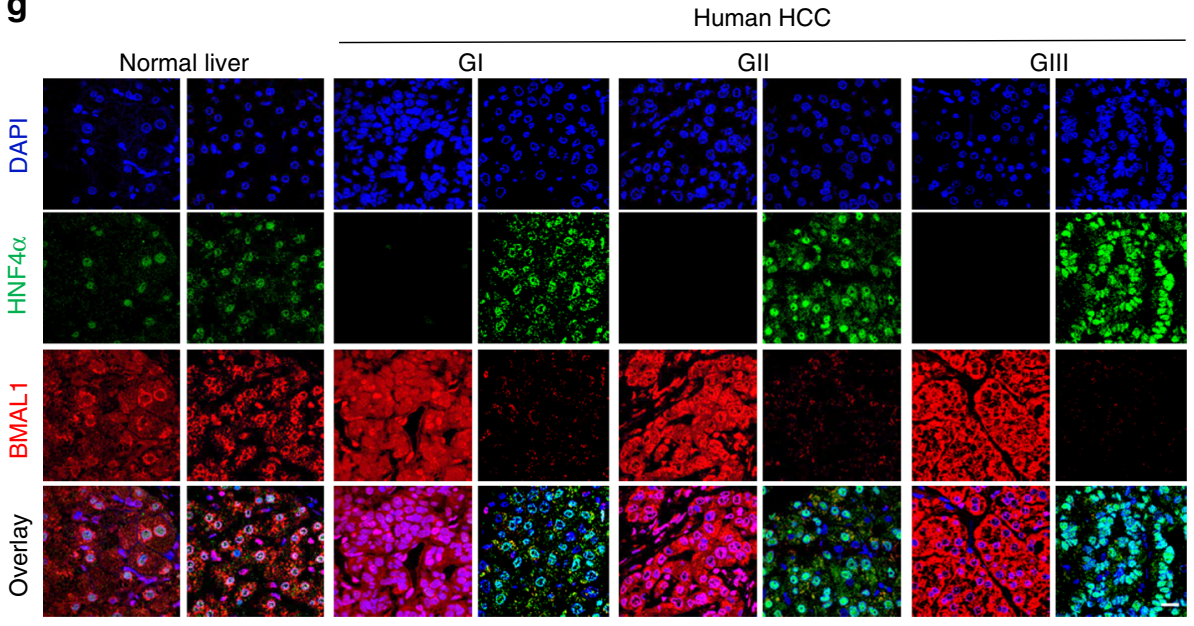

Remarkably, knockdown of only the $\mathrm{P} 2$ isoform resulted in a robust increase in BMAL1 protein, but no overall increase in cyclin gene expression, although there were differences at individual time points (Fig. 3g, h). Importantly, the loss of P1- or P2HNF4a did not significantly affect the gene expression of the
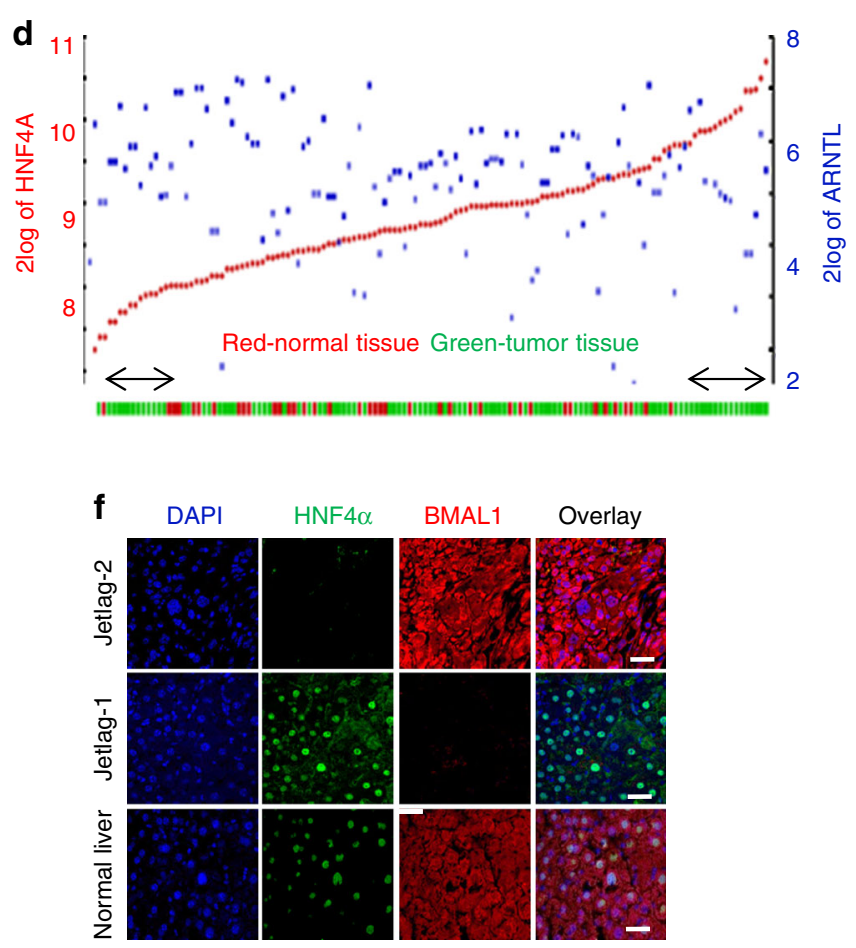

Human HCC

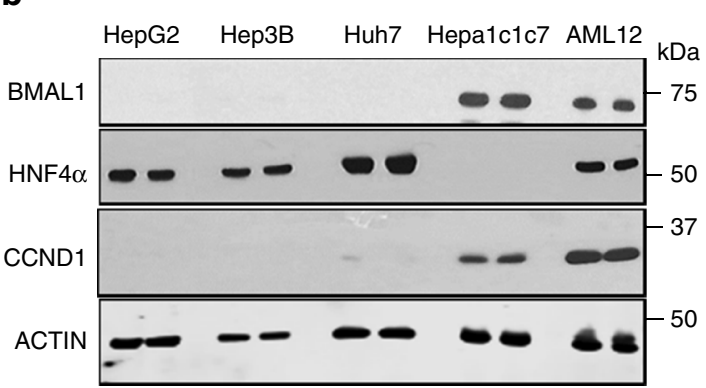

alternate isoform (Supplementary Fig. 3C). Knockdown of P2 HNF4a in SNU449, a human HCC line that exhibits only P2 expression, resulted in a similarly robust increase in BMAL1, while CCND1 was minimally affected (Fig. 3i, j). Together, these data indicate that the repressive effect of $\mathrm{P} 1-\mathrm{HNF} 4 a$ on cell cycle 
Fig. 2 Inverse expression of HNF4 $\alpha$ and BMAL1 in HCC. a RT-PCR reveals ARNTL (BMAL1) mRNA abundance in HCC and hepatoblastoma lines expressing varying levels of P1/P2-Hnf4a mRNA as well as the nontransformed hepatocyte cell line, AML12. Levels of BMAL1 mRNA expression compared to AML12 cells, ${ }^{\star \star} P<0.001,{ }^{\star \star \star \star} P<0.0001$, one-way ANOVA test, Dunnett's multiple comparisons test. $(N=4)$. b Western blot reveals BMAL1, P1/P2-HNF4 $\alpha$, and CCND1 protein levels in HNF4 $\alpha$-positive and HNF4 $\alpha$-negative liver cancer lines as well as in nontransformed AML12 cells. c Staining of 2D HCC cells and AML12 cells with antibodies to BMAL1 and to P1/P2-HNF4 $\alpha$. Overlap with DAPI nuclear stain. d Human HCC microarray datasets reveal inverse gene expression of HNF4a and ARNTL (BMAL1) mRNA in HCC specimens $(P<0.0017)(N=134)$. e Staining of 3D spheroids generated from HepG2 and Hepa1c1c7 cells with antibody to P1/P2-HNF4 $\alpha$ and BMAL1. Overlay with DAPI nuclear signal. f Staining of spontaneous mouse HCC from jet-lagged mice using antibodies to BMAL1 and P1/P2-HNF4 $\alpha$. Overlay with DAPI nuclear stain. $\mathbf{g}$ Human HCC specimens stained with antibodies for BMAL1 and P1/P2-HNF4 $\alpha$. Overlay with DAPI nuclear stain ( $G=$ tumor grade, increasing with number). Scale bar is $50 \mu \mathrm{m}$. Error bars $=\mathrm{SEM}$

genes is circadian, and ectopic expression of P1-HNF4a can induce circadian transcriptional repression in normal and HCC cells. In contrast, P2-HNF4a reduces the expression of the circadian protein BMAL1 in HCC.

Circadian control of migration and invasion by HNF4a. Part of P1-HNF4a's tumor suppressor activity has been attributed to its ability to repress genes involved in $\mathrm{EMT}^{32,52}$. To determine whether P1-HNF4a or P2-HNF4a differentially regulate EMT in transformed cells, single or double knockdown of P1-HNF4a and $\mathrm{P} 2-\mathrm{HNF} 4 a$ was performed in HepG2 cells prior to serum shock using siRNA. In contrast to AML12 cells, knockout of both P1HNF4a and P2-HNF4a concomitantly in HepG2 cells resulted in depression of $\mathrm{CDH} 1$ expression, but activation of $\beta$-catenin (CTNNB1), SNAI1, and SNAI2 (Supplementary Fig. 4b, c). Similarly, CDH1 was reduced following P1/P2-HNF4a inhibition while both phospho- and total $\beta$-catenin were increased (Supplementary Fig. 4b, c). Knockdown of only P1-HNF4a in HepG2 cells largely mimicked this result, with CTNNB1, SNAI2, and SNAI1 mRNAs being increased in abundance throughout the circadian cycle, while CDH1 expression was reduced at both the mRNA and protein levels (Fig. 4a, b). Loss of P1-HNF4a in HepG2 cells also resulted in an increase $\beta$-catenin phosphorylation (Fig. 4b), while overexpression of P1-HNF4a in Hepa-1c1c7 cells had the opposite effect (Supplementary Fig. 4a, b). In contrast, knockdown of P2-HNF4a in HepG2 and SNU449 cells revealed that $\mathrm{P} 2-\mathrm{HNF} 4 a$ has differential circadian effects on EMT genes compared to the $\mathrm{P} 1$ isoform. For example, the mRNA and protein abundance of $\mathrm{CDH} 1$ was increased at specific time points in the absence of P2-HNF4a (Fig. 4c, e), while CTNNB1 protein was moderately decreased (Fig. 4d, f). Finally, in contrast to the loss of P1-HNF4a, knockdown of P2-HNF4a reduced SNAI1 and SNAI2 mRNA (Fig. 4c, e, and Supplementary Fig. 4f).

To determine whether loss of P1-HNF4a vs. P2-HNF4a differentially affected EMT, HepG2 cells were infected with siRNA to both or single HNF4a isoforms prior to circadian synchronization and invasion assays, wherein the ability of cells to migrate through Matrigel toward a chemoattractant was measured. Knockdown of P1/P2-HNF4a resulted in an increase in the invasiveness of HepG2 cells (Fig. 4g), congruent with previously published results showing that $\mathrm{P} 1$ overexpression in cells reduces invasion ${ }^{32}$. Circadian synchronization positively affected invasion, though the reasons for this are unclear. Ectopic BMAL1 expression in HepG2 cells decreased their invasive potential, which was largely recovered by concomitant knockdown of P1/P2-HNF4a (Supplementary Fig. 4g). Overexpression of P1-HNF4a in HNF4a-deficient Hepa-1c1c7 cells produced an inverse phenotype, with cells invading less in both unsynchronized and synchronized conditions (Fig. 4h). When HepG2 cells were transfected with siRNA to only P1-HNF4a or P2-HNF4a, invasiveness of the cells was only increased after losing expression of the P1 isoform (Fig. 4i), and P1-HNF4a vs. P2-HNF4a overexpression in HNF4a-deficient Hepa-1c1c7 cells revealed a loss of invasion only after P1-HNF4a but not P2-HNF4a overexpression (Fig. 4j).

To determine whether HNF4a isoform-specific expression differentially affects cell proliferation, MTT assays (colorimetric assays designed to assess cell viability) were performed on HepG2 cells lacking both P1/P2 (Supplementary Fig. 4h) or each individual isoform (Fig. $4 \mathrm{k}$ and Supplementary Fig. 4i), or on Hepa-1c1c7 overexpressing one or the other isoform (Fig. 41 and Supplementary 4j). Knockdown of both isoforms resulted in an increase in HepG2 cell proliferation at 24- and $48 \mathrm{~h}$ (Supplementary Fig. 4h), which was due to the loss of the P1 isoform, as demonstrated by application of isoform-specific siRNAs (Fig. 4k). Overexpression of P1-HNF4a reduced the number of viable Hepa-1c1c7 cells at both time points, while overexpression of P2 had no effect (Fig. 4l). Thus, P1-HNF4a functions as a repressor of EMT and cell proliferation in HCC, but P2-HNF4a does not.

The P1 isoform of HNF4a is aberrantly localized in HCC. Based on the specific circadian expression of the P2-HNF4a isoform and its inverse pattern of expression with BMAL1 in the context of HNF4a-expressing HCC, we examined the subcellular localization P1-HNF4a and P2-HNF4a in HCC under conditions in which they are co-expressed. P1-HNF4a, but not P2-HNF4a, has previously been demonstrated to be phosphorylated directly by SRC kinase ${ }^{40}$, which leads to trafficking from the nucleus to the cytoplasm. Subcellular fractionation of AML12 cells and HCC lines revealed that while nontransformed AML12 cells express $\mathrm{P} 1-\mathrm{HNF} 4 \alpha$ only in the nuclear compartment, $\mathrm{P} 1-\mathrm{HNF} 4 \alpha$ is present in the cytoplasmic fraction of cancer cells (Fig. 5a). In contrast, P2-HNF4a resides in the nuclear and chromatin compartments of HCC cells (Fig. 5a). Based on the inverse expression of P2-HNF4a and BMAL1 in liver cancer, liver samples were attained from $\mathrm{P} 2-\mathrm{HNF} 4 \alpha$-only expressing mice $(\alpha 7 \mathrm{HMZ} \text { mice })^{50}$ to determine whether BMAL1 levels were affected in an otherwise normal liver. Fractionation of livers from a7HMZ mice revealed that BMAL1 was reduced in both the cytoplasmic and nuclear compartments, and also in whole cell lysates (Fig. 5b; for quantification, see Supplementary Fig. 5A).

To further determine the degree to which P1-HNF4a subcellular localization is impacted in HCC, human HCC was stained with antibodies specific for either the P1- or P2-HNF4a isoforms and compared to sections stained with the P1/P2HNF4a antibody. The staining revealed that while only P1$\mathrm{HNF} 4 \alpha$ is expressed in normal tissue, P2-HNF4a is predominantly expressed in HCC specimens (Fig. 5c and Supplementary Fig. 5b). While some P1-HNF4a was detected in human HCC, nuclear staining was considerably reduced compared to control tissue (Fig. 5c). To determine whether expression of P2-HNF4a can affect P1-HNF4a subcellular localization, AML12 cells were transfected with P2-HNF4a (HNF4a8) and analyzed by immunofluorescence (Supplementary Fig. 5c) and by Western blot analysis (Fig. 5d). P2-HNF4a overexpression resulted in a pronounced cytoplasmic export of P1-HNF4a, which was accentuated when cells were treated with MG132 (Fig. 5d and 
a
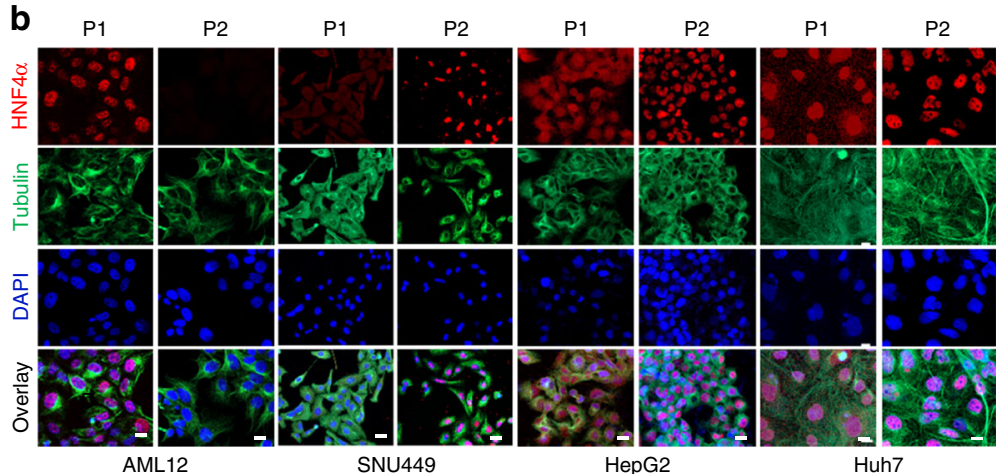

C

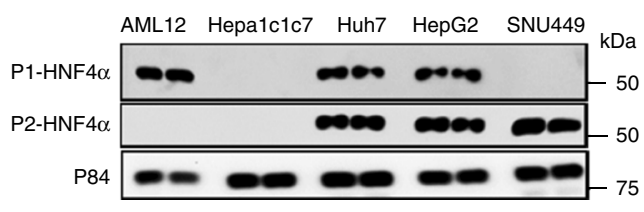

e

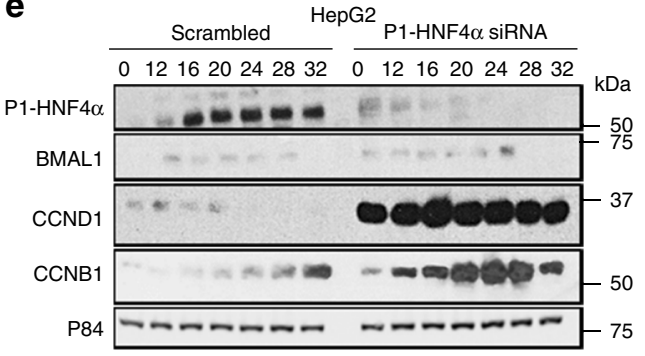

g

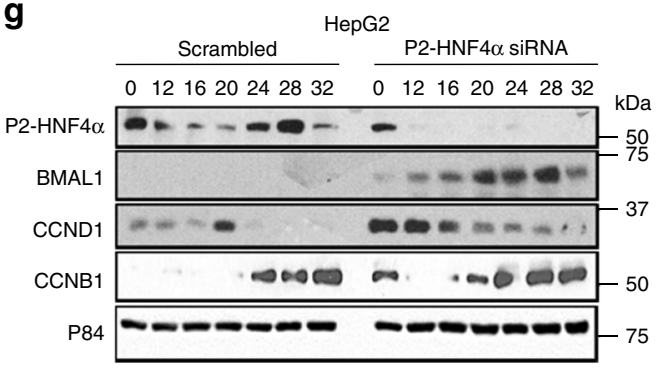

i

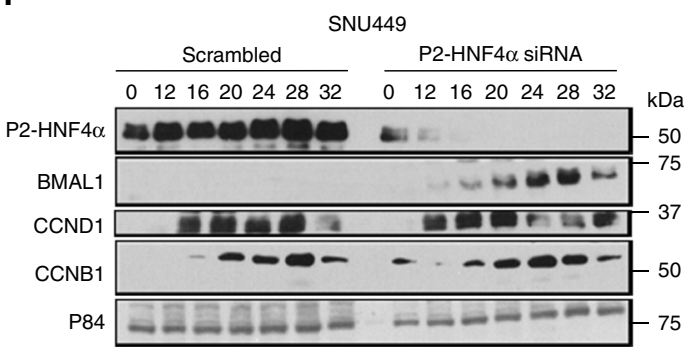

d $\leadsto$ Scrambled $\rightarrow$ P1-HNF4 $\alpha$ siRNA $\rightarrow$ Scrambled $\rightarrow$ P2-HNF4 $\alpha$ siRNA
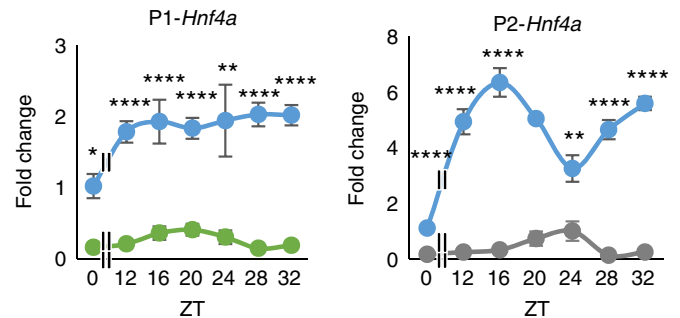

$f \rightarrow$ Scrambled $\rightarrow$ P1-HNF4 $\alpha$ siRNA
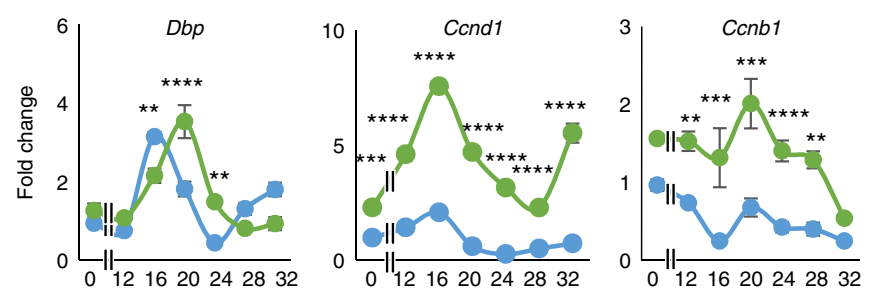

h
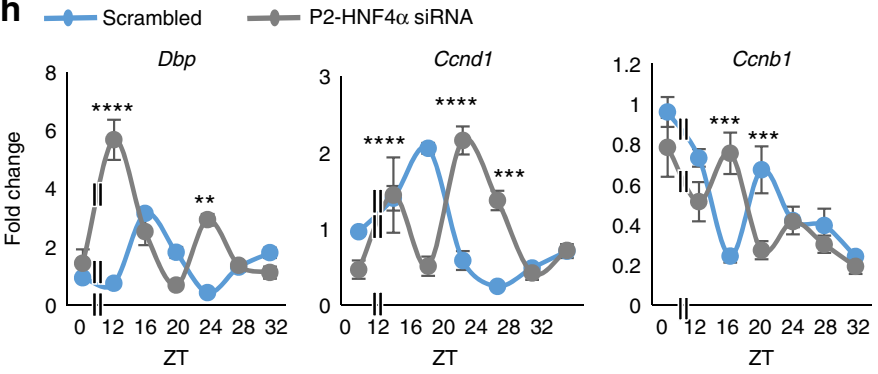

j $\rightarrow$ scrambled $\rightarrow$ P2-HNF4 $\alpha$ siRNA
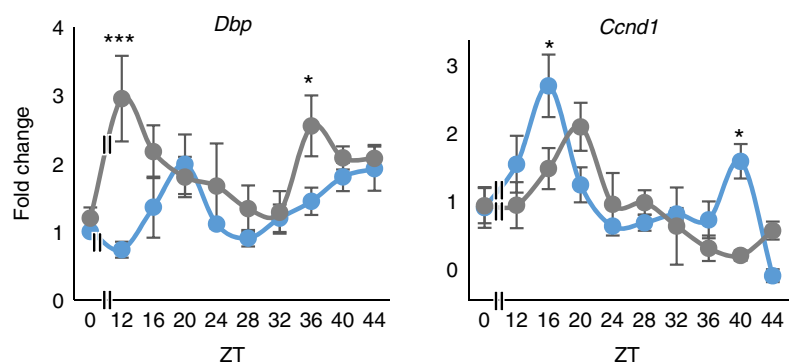

Supplementary Fig. 5c). Thus, under conditions of P1-HNF4a and P2-HNF4a co-expression, P2-HNF4a becomes the predominantly nuclear-localized isoform (Fig. 5d).

To determine whether livers expressing only $\mathrm{P} 2-\mathrm{HNF} 4 \alpha$ showed global alterations in gene expression consistent with those observed in P2-HNF4a-expressing HCC, RNA-seq was performed on WT and $\alpha 7 \mathrm{HMZ}$ livers at three different zeitgeber times. A comparison of the normalized FPKMs (Fragments Per Kilobase of transcript per Million mapped reads) revealed a pronounced upregulation of $F g f r 1$, a receptor known to be 
Fig. 3 The P2 isoform of HNF4 $\alpha$ is uniquely expressed in HCC and has distinct circadian activity. a RT-PCR reveals the mRNA abundance of P1-HNF4a and P2-HNF4a in hepatoblastoma and HCC lines, nontransformed AML12 cells, and wild-type (WT) and Hnf4a knockout (KO) liver tissues using primers to each isoform. b Staining of AML12, hepatoblastoma, and HCC cell lines with antibodies specific to P1-HNF4 $\alpha$ or P2-HNF4 $\alpha$. Overlay with DAPI nuclear stain. c Western blot of lysates from AML12, hepatoblastoma, and HCC cell lines with antibodies specific to P1-HNF4 $\alpha$, P2-HNF4 $\alpha$, or P84 proteins. d RTPCR reveals expression of P1-HNF4a or P2-HNF4a following serum synchronization in HepG2 cells and using siRNA specific to P1-HNF4a (left panel) or P2HNF4a (right panel). e Western blot analysis showing P1-HNF4 $\alpha$, BMAL1, CCND1, and CCNB1 protein expression in HepG2 cells serum shocked and previously treated with siRNA specific to P1-HNF4a or with scrambled oligonucleotides. $f$ RT-PCR reveals the expression of DBP, CCND1, and CCNB1 following serum shock and knockdown of P1-HNF4a with specific siRNA or scrambled (Sc) oligonucleotides. $\mathbf{g}$ Western blot showing P2-HNF4 $\alpha$, BMAL1, CCND1, and CCNB1 protein expression following expression of scrambled or P2-HNF4 $\alpha$-specific siRNA oligonucleotides. $\mathbf{h}$ RT-PCR reveals the circadian expression of DBP, CCND1, and CCNB1 following knockdown of P2-HNF4 $\alpha$. i Western blot showing P2-HNF4 $\alpha$, BMAL1, CCND1, and CCNB1 expression in SNU449 cells after serum shock and previously treated with scrambled oligonucleotides or siRNA oligonucleotides specific to P2-HNF4 $\alpha$. $\mathbf{~ R T}$-PCR reveals the expression of DBP and CCND1 following the application of scrambled (Sc) or siRNA specific to the P2-HNF4a isoform in SNU449 cells. Two-way ANOVA, Sidak's multiple comparisons test, ${ }^{\star} P<0.03,{ }^{\star \star} P<0.005,{ }^{{ }^{\star \star \star}} P<0.0005,{ }^{\star \star \star \star} P<0.0001 .(N=4)$. Scale bar $50 \mu \mathrm{m}$. (See Supplementary Table 1 for JTK_Cycle Rhythmicity Statistics.) Error bars = SEM

associated with HCC progression and metastasis ${ }^{53}$ (Fig. 5e). Similarly, the matrix metallopreoteinase $M m p 14$, whose transcriptional regulation by HNF4a has been reported to be involved in metastasis ${ }^{54}$, was increased at several time points in a7HMZ mice. a7HMZ livers showed a downregulation of $C d h 1$ at some time points, suggesting an altered circadian phase in the absence of P1-HNF4a. Interestingly, expression of Src was elevated in a7HMZ livers, which could in part explain the nuclear export of $\mathrm{P} 1-\mathrm{HNF} 4 a$ in P2-HNF4a-positive $\mathrm{HCC}^{40}$. Finally, Myc abundance was increased in P2-HNF4a expressing livers, consistent with increased proliferation of HCC expressing the P2 isoform.

HNF4a has previously been linked to the $\mathrm{Wnt} / \beta$-catenin pathway and the isoforms show distinct recruitment to genes involved in $\mathrm{Wnt} / \beta$-catenin signaling ${ }^{30}$. To examine whether Wnt/ $\beta$-catenin signaling might be different in $\alpha 7 \mathrm{HMZ}$ livers, RNA-seq data was analyzed for changes in expression of genes involved in this pathway. Several $\mathrm{Wnt} / \beta$-catenin pathway genes were significantly altered between WT and a7HMZ livers, with a pronounced upregulation of the positive regulator Porcn in a7HMZ livers, but a downregulation of several negative regulators of the pathway, including Ctnnbip1, Serpinf1, and Axin1 (Fig. 5f). Interestingly, Serpinf1 (also known as Pedf) has been shown to negatively regulate the $\mathrm{Wnt} / \beta$-catenin pathway in the liver specifically ${ }^{55}$ and is reported to have antiangiogenic activity in the context of HCC $^{56}$. Thus, downregulation of Serpinf1 in the context of a7HMZ livers is consistent with P2HNF4a activation of the $\mathrm{Wnt} / \beta$-catenin pathway and promoting tumor growth.

Direct and indirect repression of BMAL1 by P2-HNF4a. To elucidate what mechanisms might be responsible for the incompatibility of BMAL1 and HNF4a in HCC, we determined whether HNF4a regulates the transcription of the BMAL1 promoter. Hepa-1c1c7 cells were transiently transfected with P1- or P2HNF4a expression constructs and a BMAL1-LUC reporter with or without the BMAL1 activator, retinoic acid receptor related orphan receptor A (RORa). Because prior studies have revealed that MYC can repress BMAL1 expression in specific cancer cells ${ }^{14,57}$, we co-transfected the cells with siMyc or scrambled oligonucleotides to determine the extent to which HNF4a repression of Bmallcould be indirect through changes in $M y c$ expression. While both P1- and P2-HNF4a reduced basal and ROR $\alpha$-activated BMAL1-driven LUC expression, P2-HNF4a had a much stronger repressive effect, reducing the ROR $\alpha$-induced activation to near baseline levels (Fig. 6a). Compared to scrambled oligonucleotides, the addition of siMyc contributed a further $\sim 16$ and $7 \%$ reduction over $\mathrm{P} 1-\mathrm{HNF} 4 \alpha$ and $\mathrm{P} 2-\mathrm{HNF} 4 \alpha$ repression of Bmal1-luc, respectively (Supplementary Fig. 6A). ChIP-seq reveals that P1-HNF4a binds the murine Bmal1 promoter at approximately 350 base pairs upstream of the transcriptional start site (Supplementary Fig. 6b). To confirm whether the distinct isoforms of HNF4a interact with the ARNTL locus in normal and cancer cells, HNF4a ChIP was performed on HepG2 cell extracts (Fig. 6b and Supplementary Fig. 6d) and mouse liver (Supplementary Fig. 6c). Antibodies specific to each isoform revealed that P2-HNF4a shows superior binding to ARNTL in human HepG2 cells (Fig. 6b). ChIP of normal liver using the P1/P2-HNF4a antibody confirmed that P1-HNF4a can also bind to the Arntl promoter (Supplementary Fig. 6c). (For ChIP in HepG2 cells using the P1/P2-HNF4a antibody, see Supplementary Fig. 6d). Thus, HNF4a both binds the BMAL1 promoter and represses BMAL1 expression at the transcriptional level, with the P2 isoform providing the primary nuclear repression in HCC cells (Fig. 5b-e). Thus, we conclude that while $M y c$ upregulation in response to loss of nuclear P1-HNF4a may contribute marginally to BMAL1 loss in HCC, the primary mechanism involves direct transcriptional repression by $\mathrm{P} 2-\mathrm{HNF} 4 \alpha$.

Ectopic BMAL1 in HNF4a-positive HCC impairs tumor growth. To determine whether forced expression of BMAL1 in BMAL1-deficient, HNF4a-positive hepatoblastoma and HCC impairs tumor growth, AML12, HepG2, Huh7, and SNU449 cells were transfected with an expression vector for BMAL1 and analyzed for growth and viability. Ectopic expression of BMAL1 in HepG2 cells resulted in a transient loss of HNF4a expression (Supplementary Fig. 6h); and cancer cells, but not AML12 cells overexpressing BMAL1, showed reduced proliferative capacity over 48 h. (Supplementary Fig. 6e). Furthermore, overexpression of P1-HNF4a in Hepa-1c1c7 cells significantly impaired the ability of cells to form 3D spheroids after 10 days in Matrigel, as did the transient transfection of GFP-Bmall in HepG2 cells (Supplementary Fig. 6f-g). To determine whether the growth of liver tumors in vivo could be impeded by ectopic BMAL1 expression, we injected immune compromised NSG (NOD.CgPrkdc scid $I l 2 r g^{t m 1 W j l} / \mathrm{SzJ}$ ) mice subcutaneously with HepG2 or SNU449 cells stably expressing luciferase and co-transfected with GFP-BMAL1 or empty vector. Bioluminescence was measured on day 0 to ensure similar cell numbers were injected, and tumor growth was monitored weekly for 4 weeks. Compared to cells expressing luciferase only, BMAL1-expressing HepG2 and SNU449 cells were significantly retarded in tumor growth, resulting in a complete lack of tumor growth or substantially smaller tumors (Fig. 6c and Supplementary Fig. 6i). Thus, we conclude that the co-expression of P2-HNF4a and BMAL1 in HCC is incompatible with tumor cell proliferation in vitro and in vivo.

To determine the mechanisms by which BMAL1 induces cell death in HNF4a-positive cancer cells, HepG2 and SNU449 cells 
a $\longrightarrow$ Scrambled $\longrightarrow$ P1-HNF4 $\alpha$ siRNA
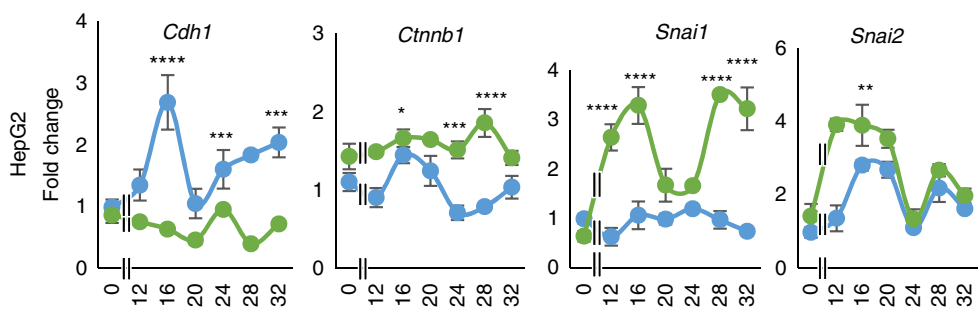

C
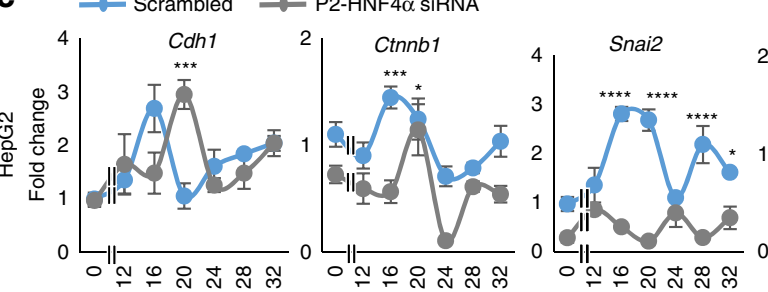

e

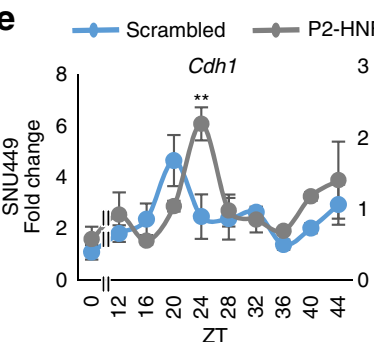

g

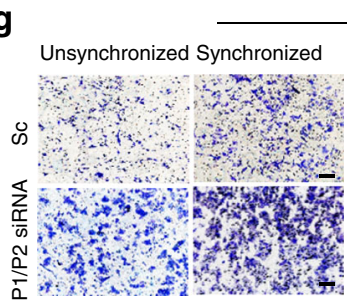

HepG2

$\square$ Scrambled $\boxminus \mathrm{P} 1 / \mathrm{P} 2$ siRNA

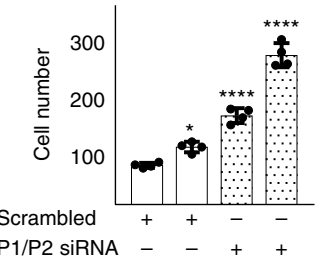

Serum shock +-++

i
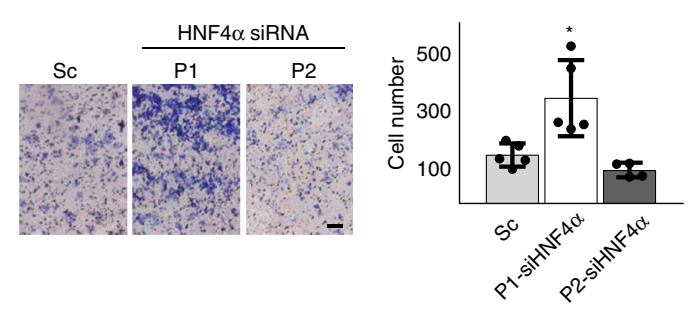

$\mathbf{k}$

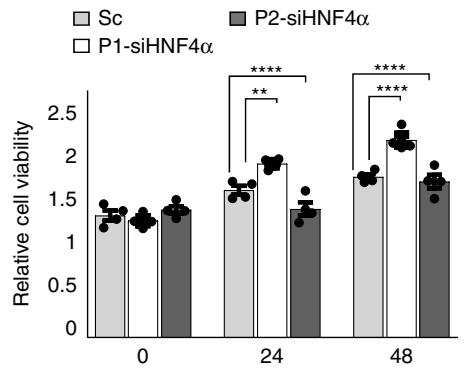

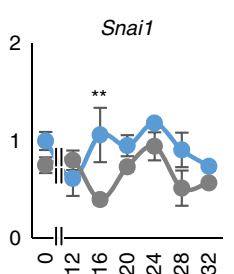

b

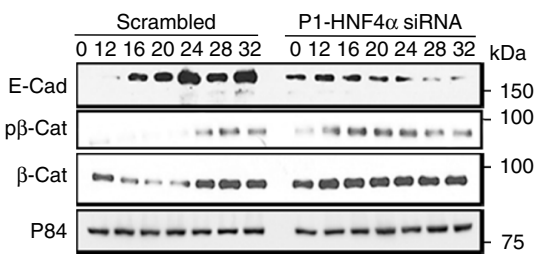

d Scrambled P2-HNF4 $\alpha$ siRNA

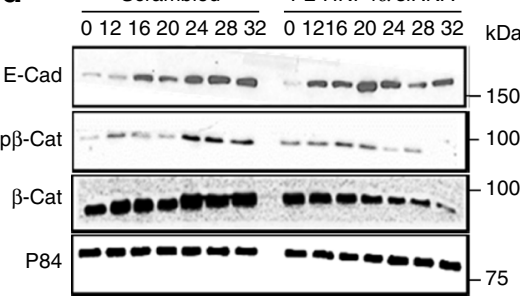

f

Scrambled $\quad \mathrm{P} 2-\mathrm{HNF} 4 \alpha$ siRNA

$0121620242832 \quad 0121620242832 \mathrm{kDa}$

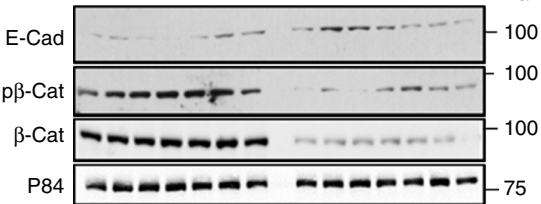

Hepa1c1c7

h

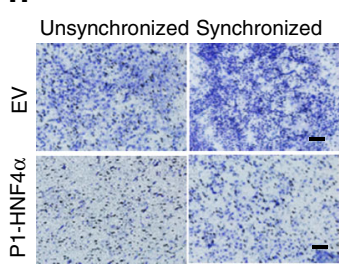

$\square \mathrm{EV} \quad \mathrm{P} 1-\mathrm{HNF} 4 \alpha$

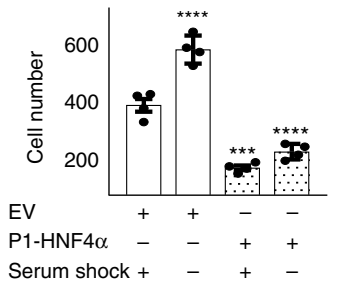

j
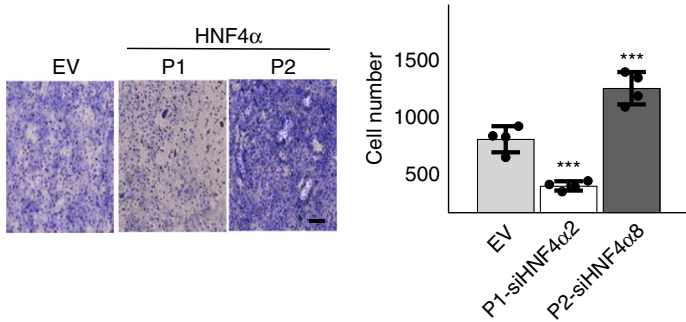

I $\square$ EV $\square$ P2-HNF4 88

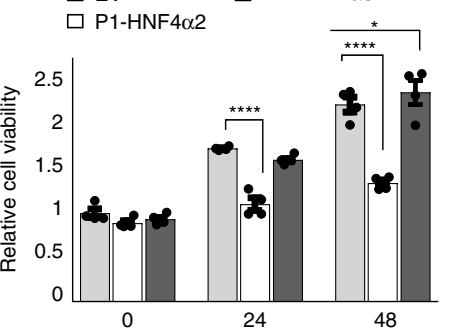

were transfected with GFP or GFP-BMAL1. Expression of GFPBMAL1 resulted in the induction of the tumor suppressor P53 as well as cleaved caspase 3 protein at all circadian time points tested following serum shock (Fig. 6d). Staining of unsynchronized cells transfected with GFP or GFP-BMAL1 revealed an increase in cleaved caspase 3 in BMAL1 overexpressing cells (Fig. 6e), suggesting that forced expression of the circadian protein BMAL1 in HNF4a-positive HCC inhibits tumor growth by inducing apoptosis, though additional BMAL1-mediated mechanisms may also contribute ${ }^{57,58}$. 
Fig. 4 Circadian control of EMT by HNF4 $\alpha$ is isoform specific. a RT-PCR reveals mRNA abundance of EMT genes CDH1, CTNNB1, SNAI1, and SNAI2 after serum shock with prior application of scrambled oligonucleotides or siRNA specific to P1-HNF4 $\alpha$ in HepG2 cells. b Western blot showing expression of $\mathrm{CDH} 1$, phosphorylated, and total $\beta$-catenin (CTNNB1, $\beta$-Cat), after P1-HNF4 $\alpha$ knockdown followed by serum shock. c RT-PCR reveals expression of EMT genes following serum shock and prior application of scrambled or siRNA specific to P2-HNF4a. d Western blot showing expression of CDH1, phosphorylated and total $\beta$-Cat following knockdown of P2-HNF4 $\alpha$ and serum shock. e RT-PCR reveals circadian expression of EMT genes CDH1, CTNNB1, and SNAl1 after inhibition of P2-HNF4 $\alpha$ using specific siRNA oligonucleotides in SNU449 cells. $\mathbf{f}$ Western blot showing the expression of CDH1 and phosphorylated and total $\beta$-Cat after P2-HNF4 $\alpha$ knockdown following serum shock in SNU449 cells. Two-way ANOVA, Sidak's multiple comparisons test, ${ }^{\star} P<0.03,{ }^{\star \star} P<0.005,{ }^{\star \star \star} P<0.0005,{ }^{\star \star \star \star} P<0.0001,(N=4)$. $\mathbf{g}$ Invasion assay reveals invaded unsynchronized or circadian synchronized HepG2 cells expressing scrambled or siRNA for P1/P2-HNF4a, $48 \mathrm{~h}$ after plating. Quantification, right panel. $\mathbf{h}$ Invaded unsynchronized or synchronized Hepa-1c1c7 cells following serum synchronization and prior overexpression of P1-HNF4 $\alpha$. Quantification, right panel. $\mathbf{i}$ Invaded synchronized HepG2 cells following application of scrambled ("Sc"), P1-HNF4a, or P2-HNF4a siRNA oligonucleotides. Quantification, right panel. $\mathbf{j}$ Invaded synchronized Hepa-1c1c7 cells following overexpression of P1-HNF4 $\alpha$ or P2-HNF4 $\alpha$. Quantification, right panel. Compared to SC or EV at the same time: ${ }^{\star} P<0.05,{ }^{\star \star} P<0.01,{ }^{\star \star \star} P<0.001$, ${ }^{\star \star \star \star} P<0.0001$, one-way ANOVA test, Dunnett's multiple comparisons test. $(N=5)$. k Fold change in proliferating HepG2 cells following P1-HNF4 $\alpha$ vs. P2HNF4 $\alpha$ knockdown at 24- and $48 \mathrm{~h}$ using MTT assay. I MTT assay reveals proliferating Hepa-1c1c7 cells after transfection with empty vector (EV), P1$\mathrm{Hnf} 4 a(\mathrm{Hnf} 4 \mathrm{a} 2)$ or P2-Hnf4a (Hnf4a8) at 24- and $48 \mathrm{~h}$ using MTT assay. Comparing SC/EV to P1/P2-siHNF4 $\alpha$ or between P1-siHNF4 $\alpha$ and P2-siHNF4 $\alpha$ : Two-way ANOVA, Sidak's multiple comparisons test, ${ }^{\star} P<0.03,{ }^{\star \star} P<0.005,{ }^{\star \star \star} P<0.0005,{ }^{\star \star \star \star} P<0.0001,(N=6)$. Scale bar $100 \mu$ m. (See Supplementary Table 1 for JTK_Cycle Rhythmicity Statistics.) Error bars = SEM

\section{Discussion}

Our results reveal that the P1 and P2 isoforms of HNF4a have distinct circadian roles. In addition, they show that, as in colon cancer, $\mathrm{P} 1-\mathrm{HNF} 4 \alpha$ is tumor suppressive, while $\mathrm{P} 2-\mathrm{HNF} 4 \alpha$ is not. These data provide a model by which the upregulation of P2HNF4a is causal for downregulation of BMAL1 expression in human HCC, consistent with findings in exon swap mice expressing P2-HNF4a in normal liver (Fig. 5). Furthermore, these data reveal that forced expression of BMAL1 inhibits HNF4apositive tumor growth (Fig. 7). Taken together, these results suggest that targeting the circadian clock in HCC may be a promising treatment for the growth and progression of HCC tumors.

Several interesting scenarios regarding P2-HNF4a expression in HCC are plausible. Firstly, in spontaneous human HCC, P2HNF4a is selectively induced ${ }^{39}$ by yet unidentified mechanisms. Interestingly, the proto-oncogene SRC can phosphorylate and down-regulate P1-HNF4a. Because P1-HNF4a represses the P2 promoter $^{30}$, elevated SRC could potentially be leading to the induction of P2-HNF4a in HCC. SRC has been found to be overexpressed in HCC, and SRC inhibitors are a first-line chemotherapeutic treatment for liver cancer, although some patients are refractory to the treatment ${ }^{59}$. Our data suggest that P2HNF4a increases SRC expression, which may provide a positive feedback loop in HCC.

Since P2-HNF4a expression results in an increase in cytoplasmic P1-HNF4a (as does SRC-induced phosphorylation), circadian repression in the nucleus of $M Y C, C C N D 1, C C N D 1$, among other genes normally repressed in a healthy liver by P1HNF4a appears to be reduced in HCC. However, ectopic expression of $\mathrm{P} 1-\mathrm{HNF} 4 \alpha$ in HCC can still produce circadian repression of these targets, and knockdown of $\mathrm{P} 1-\mathrm{HNF} 4 \mathrm{a}$ in $\mathrm{HNF} 4 a$-positive HCC can increase the expression of these targets. In addition to targeting HCC by increasing BMAL1-mediated clock function, HCC growth could potentially be prevented by inhibiting P1-HNF4a from exiting the nucleus.

While HNF4a and BMAL1 are robustly co-expressed in normal hepatocytes, of significance is the distinct downregulation of BMAL1, which has been demonstrated to play tumor suppressive roles ${ }^{14,57,58}$ in HNF4a-positive HCC. The data presented here reveal that $\mathrm{P} 2-\mathrm{HNF} 4 \alpha$ contributes to this by providing strong transcriptional repression of the Bmall gene. While P1-HNF4a has a modest ability to repress Bmal1 in luciferase assays in vitro, $\mathrm{P} 2-\mathrm{HNF} 4 a$ provides much stronger repression of Bmall and furthermore, appears to be the primary nuclear-localized isoform in the context of HCC (see Fig. 5). Preferential binding of P2-
HNF4a over P1-HNF4a to one or more corepressor proteins may contribute to greater repression of BMAL1. Consistent with what has been observed in the colon ${ }^{28}$, we find that the P1-HNF4a isoform preferentially binds the nuclear receptor ESRRA (Supplementary Fig. 7c). On the other hand, P2-HNF4a appears to preferentially bind PROX1 (Supplementary Fig. 7c), a protein known to interact with the HDAC3 repressive complex ${ }^{60}$. However, we have not yet confirmed whether these isoform-specific interactions contribute to differential repression of BMAL1. It is also conceivable that $\mathrm{P} 2-\mathrm{HNF} 4 a$ preferentially interacts with chromatin modifiers or with other transcriptional repressors that result in repression at specific target genes.

HCC appears to fall into two categories as it relates to HNF4a expression: either P2-HNF4a is induced so that the cells express both P1-HNF4a and P2-HNF4a or only the P2 isoform, or tumors are devoid of HNF4a expression altogether. At least for HNF4a-positive HCC, these data reveal the therapeutic potential of targeting the circadian clock in tumor progression. For example, there are known molecules that enhance clock function directly through the activation of a specific BMAL1 transcriptional regulator ${ }^{61}$, and thus it is worth determining whether such molecules might be employed as interventions in HNF4a-positive HCC. Furthermore, specific ligands have been proposed for HNF4a $\alpha^{62-64}$. HNF4a has been shown to bind its endogenous ligand, linoleic acid, in a reversible fashion, indicating that $\mathrm{HNF} 4 \alpha$ is a potential drug target ${ }^{63}$. Circadian targeting of normal vs. HCC cells with such ligands could provide a unique opportunity for tumor prevention or treatment if compounds that act in an isoform-specific fashion could be identified.

While HCC incidence in humans has historically been associated with viral infections, emerging evidence identifies nonalcoholic fatty liver disease and nonalcoholic steatohepatitis as major contributors to the increase in $\mathrm{HCC}^{65,66}$. Interestingly, we find an upregulation in the expression of the P2 isoform in mice chronically fed a high-fat diet (HFD) (Fig. $6 \mathrm{f}$ and Supplementary Fig. 7a), as well as a partial redistribution of the P1 isoform from the nucleus to the cytoplasm. Similarly, we find an induction of the $\mathrm{P} 2$ isoform in the livers of $d b / d b$ mice, which are known to exhibit steatosis and fibrosis (Supplementary Fig. 7b). This suggests that aberrant expression of P2-HNF4a may be involved in the pathogenesis of HCC. Importantly, circadian disruption mimicking human jet-lag in mice induces spontaneous HCC via the development of fatty liver ${ }^{17}$, and we consider circadian disruption in humans to be an inadequately studied link to the increase in HCC, but of considerable translational value. Thus, circadian targeting of HCC holds great promise and may have 
a
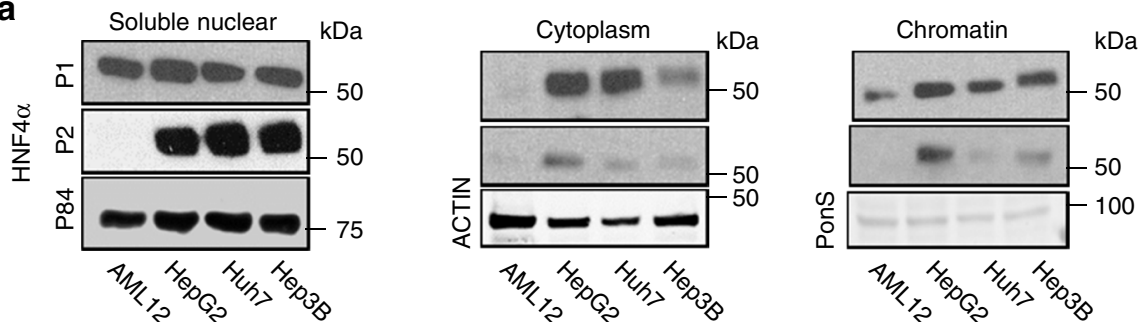

b
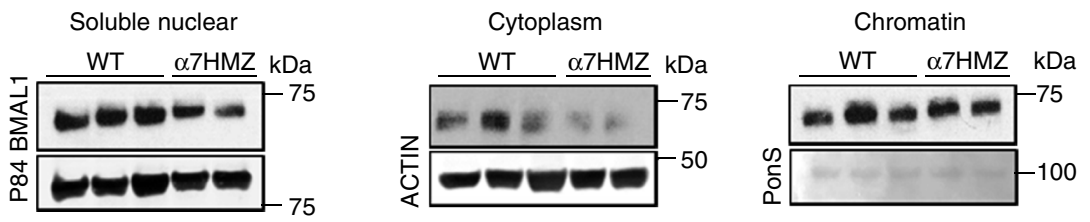

Whole cell lysate

C
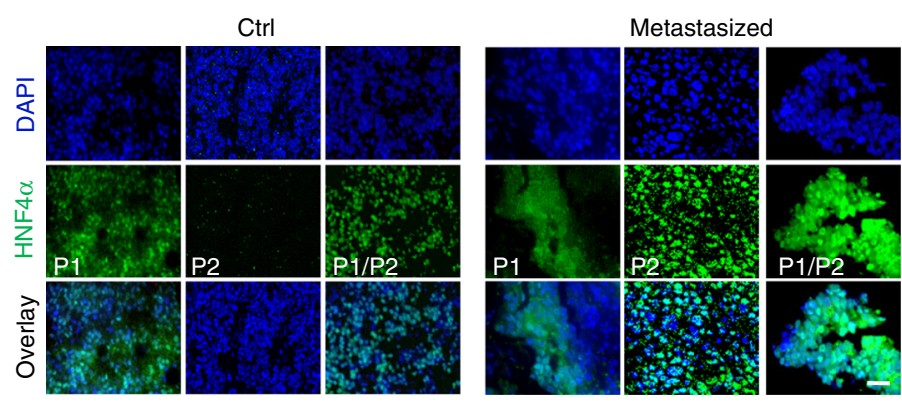

e 口WT $\square$ a $\mathrm{HHMZ}$
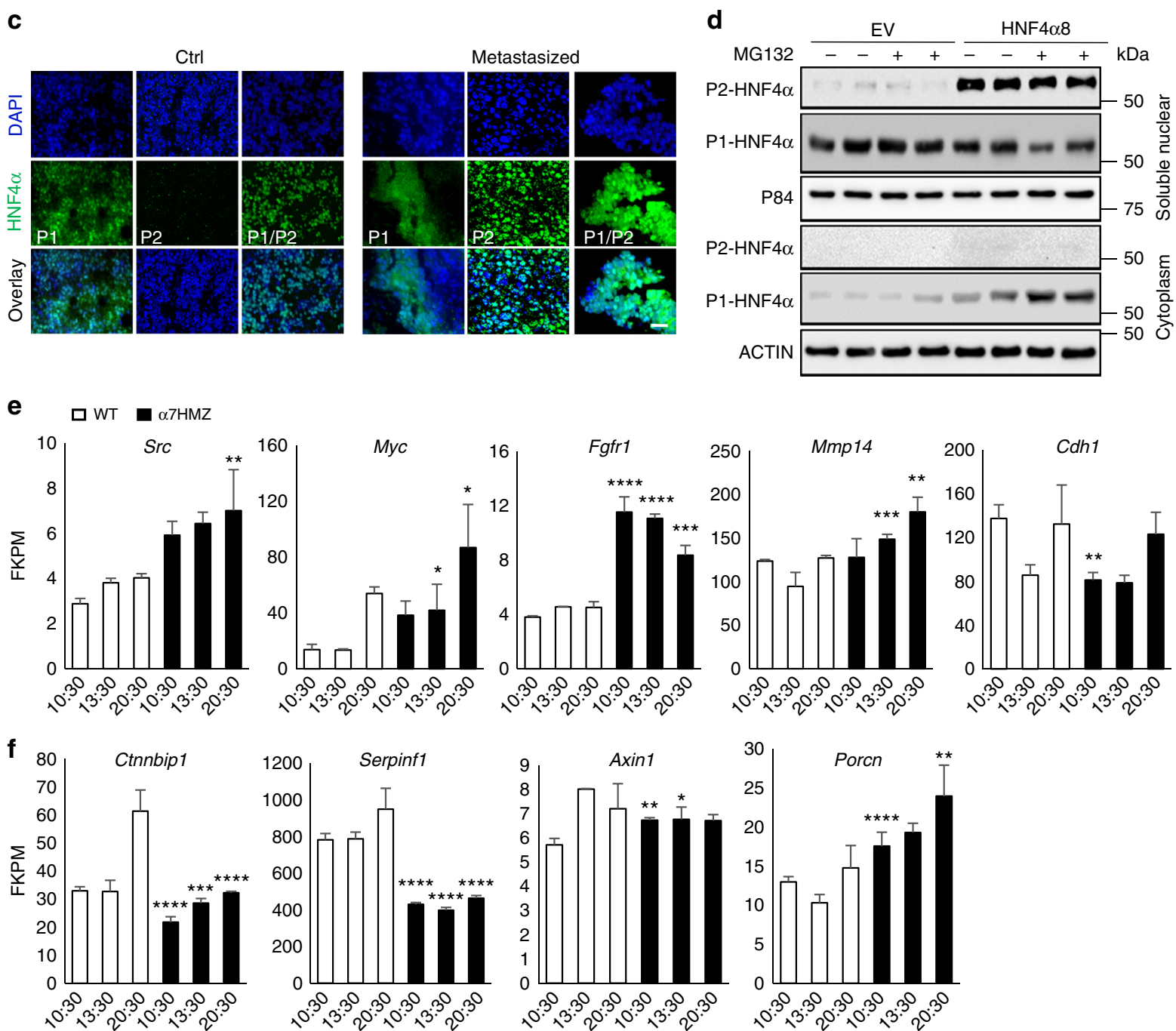

Fig. 5 Altered subcellular localization of HNF4 $\alpha$ isoforms in HCC. a Western blot shows the expression of P1-HNF4 $\alpha$ and P2-HNF4 $\alpha$ in the soluble nuclear, cytoplasmic, and chromatin fractions of AML12, hepatoblastoma, and HCC lines. b Western blot showing the expression of BMAL1 in cellular compartments and whole cell lysates of mice expressing exclusively P1-HNF4 $\alpha$ (WT) or P2-HNF4 $\alpha(\alpha 7 \mathrm{HMZ}$ mice) (PonS = ponceau S).

c Immunofluorescence of control tissue and metastasized human HCC specimens with antibodies specific to P1-HNF4 $\alpha$, P2-HNF4 $\alpha$, or to P1/P2-HNF4 $\alpha$ d Western blot showing P2-HNF4 $\alpha$ and P1-HNF4 $\alpha$ localization to the soluble nuclear compartment or the cytoplasm following overexpression of P2HNF4 $\alpha$ in AML12 cells. e FKPM counts from RNA-seq for Src, Myc, Fgfr1, Mmp14, and Cdh1 mRNAs in WT or $\alpha$ HMZ livers. f FKPM counts from RNA-seq for Ctnnbip1, Serpinf1, Axin1, and Porcn in WT or $\alpha \mathrm{HMZ}$ livers. ${ }^{\star} P<0.05,{ }^{{ }^{*}} P<0.01,{ }^{\star \star \star} P<0.001,{ }^{\star \star \star \star} P<0.0001,(N=3)$. Scale bar is $50 \mu \mathrm{m}$. Error bars $=$ SEM 
a

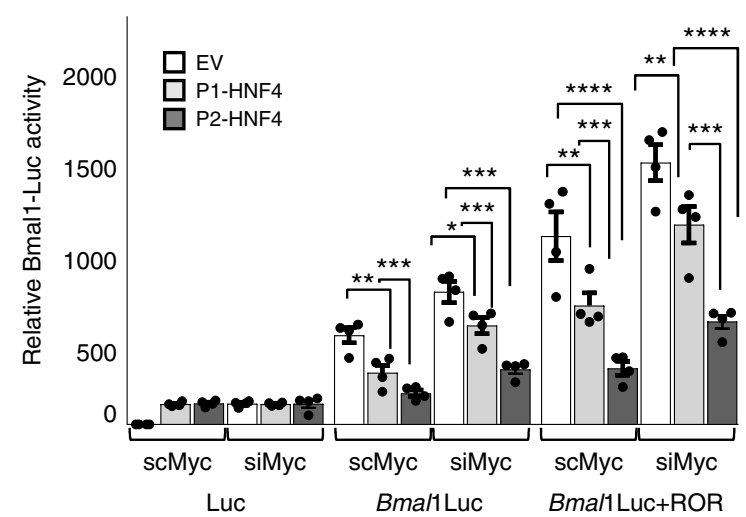

C

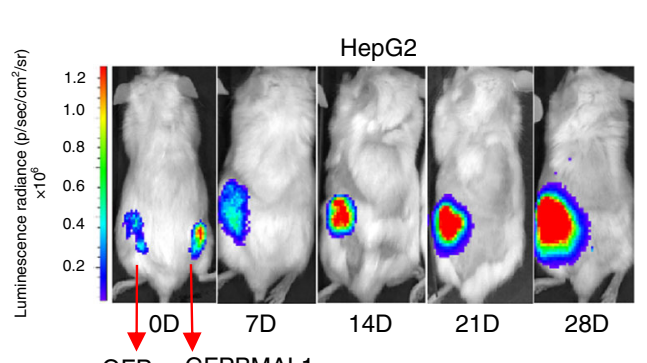

GFP- GFPBMAL1-

LUC LUC

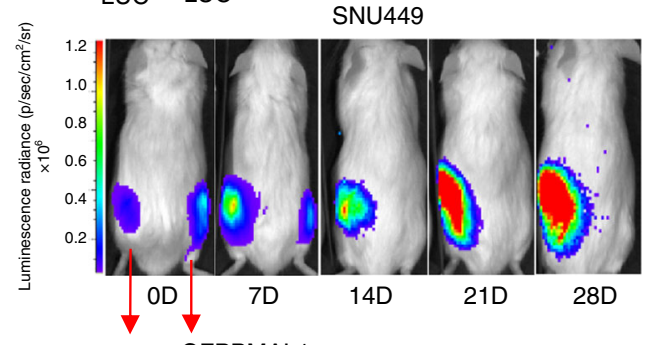

GFP- GFPBMAL1-

LUC LUC b

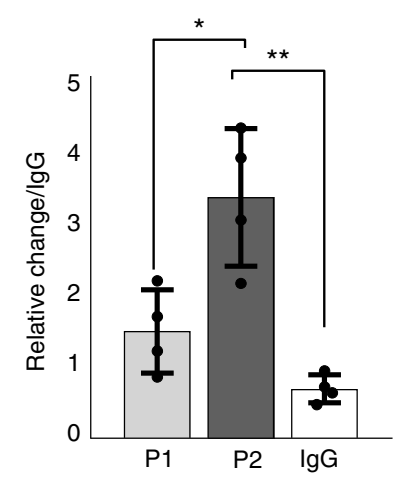

d
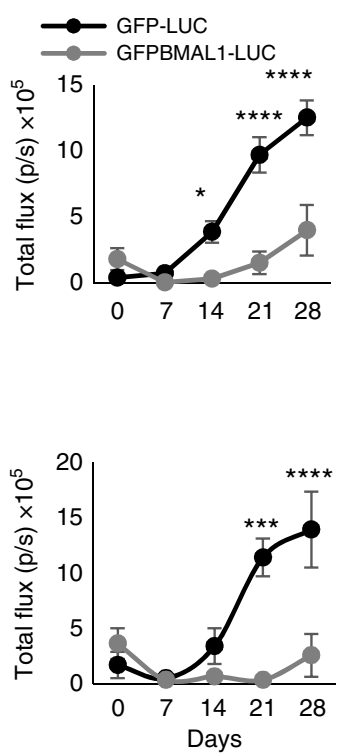

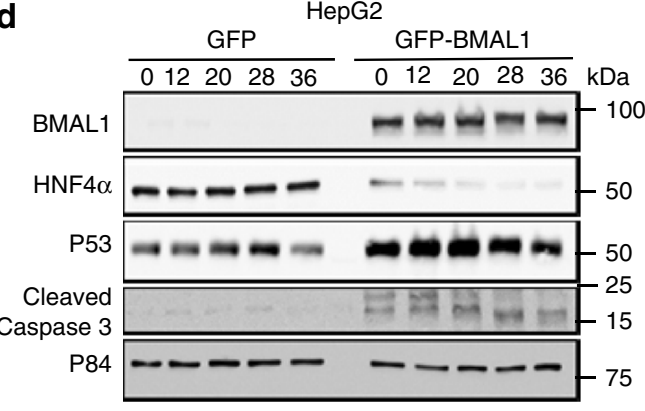

SNU449

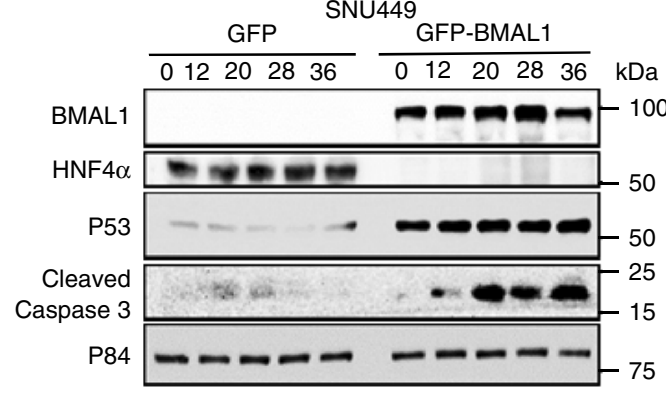

e

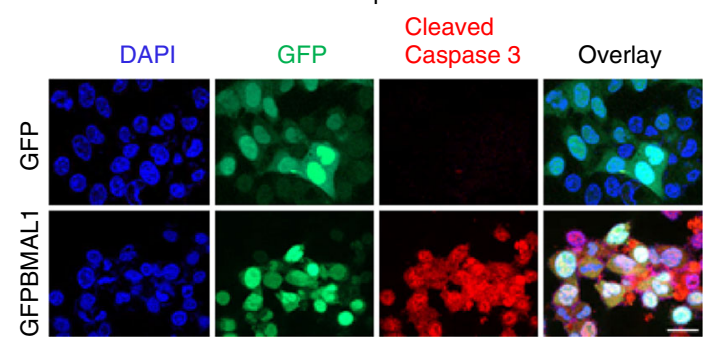

SNU449

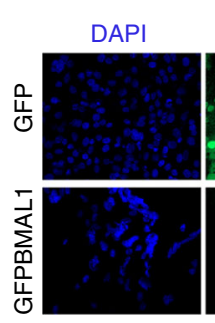

Cytoplasm

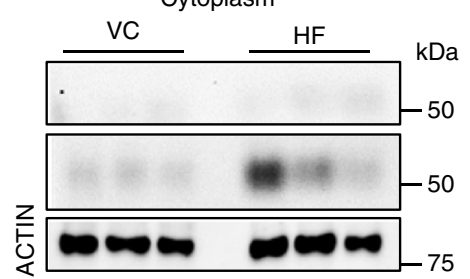

Cleaved
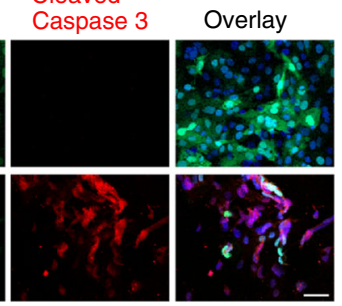

f

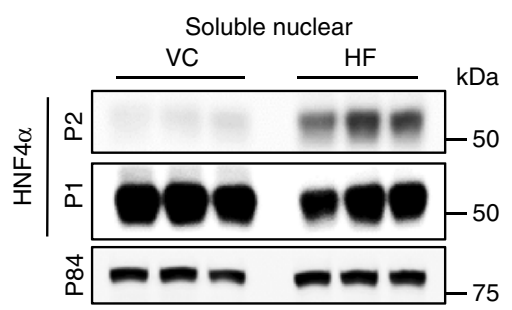

Whole cell lysate

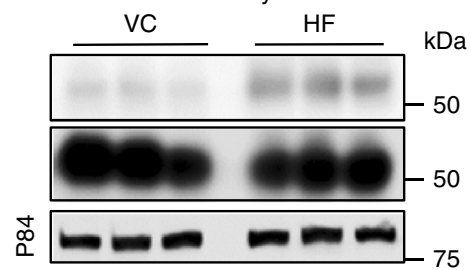

advantages over traditional therapies due to decreased toxicity in hepatic and extra-hepatic tissues. Future studies investigating the clinical utility of circadian intervention in HCC are essential and highly promising.

\section{Methods}

Cell culture. Cell lines were obtained from the American Tissue Culture Collection (ATCC, Manassas, VA). SNU449, HepG2, Huh7, Hep3B, and Hepa1clc7 cells were grown in Eagle's Minimal Essential Medium, and AML12 cells were grown in a 1:1 mixture of Dulbecco's Modified Eagle's Medium and Ham's 
Fig. 6 BMAL1 overexpression in HNF4 $\alpha$-positive HCC inhibits tumor growth a Luciferase assay results showing luciferase expression from BMAL1-LUC following transfection of DNA for the empty vector (EV), P1-Hnf4a or P2-Hnf4 $\alpha$ with or without co-expression of ROR $\alpha$ and with co-application of scrambled or Myc siRNA oligonucleotides. Comparing EV to P1/P2-HNF4 $\alpha$. Two-way ANOVA, Sidak's multiple comparisons test, ${ }^{\star} P<0.03,{ }^{\star \star} P<0.005$, ${ }^{\star \star \star} P<0.0005,{ }^{\star \star \star \star} P<0.0001,(N=6)$. b Chromatin immunoprecipitation (ChIP) of P1- or P2-HNF4 $\alpha$ in HepG2 cells followed by qPCR reveals amplification of BMAL1 sequence upstream of the transcriptional start. $\mathbf{c}$ In vivo bioluminescence of hepatoblastoma and HCC tumors in immune compromised mice on days $0,7,14,21$, and 28 after subcutaneous injection of HepG2 or SNU449 cells expressing vectors Luc and Gfp or Luc and GfpBmal1. Quantification of tumor size, right panel. Two-way ANOVA, Sidak's multiple comparisons test, ${ }^{\star} P<0.03,{ }^{\star \star} P<0.005,{ }^{\star \star \star} P<0.0005,{ }^{\star \star \star \star} P<$ 0.0001. $(N=6-9)$. Scale bar is $100 \mu \mathrm{m}$. d Western blot reveals the abundance of BMAL1, P1/P2-HNF4 $\alpha$, P53, cleaved caspase 3, and P84 after serum synchronization of HepG2 and SNU449 cells previously transfected with Gfp or Gfp-Bmal1. e Staining of HepG2 and SNU449 cells 48 h after infection with virus containing Gfp-Bmal1, using antibodies to GFP and cleaved caspase 3. Overlay with DAPI nuclear stain. Scale bar is $20 \mu \mathrm{m}$. $\mathbf{f}$ Western blot showing P2HNF4 $\alpha$ and P1-HNF4 $\alpha$ localization in the soluble nuclear and cytoplasmic cellular compartments, or in whole cell lysates of livers from animals with dietinduced obesity, using antibodies specific to P2-HNF4 $\alpha$ or P1-HNF4 $\alpha$. Error bars $=$ SEM

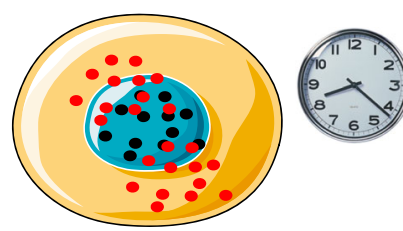

HNF $4 \alpha^{+}$hepatocyte
1. P1-HNF4 $\alpha$ expressed only in the nucleus

2. Circadian restrain of $M Y C$ and cyclin gene expression

3. Co-expression of BMAL1 and P1$\mathrm{HNF} 4 \alpha$

\section{- P1-HNF4a \\ - BMAL1 \\ P2-HNF4 $\alpha$}

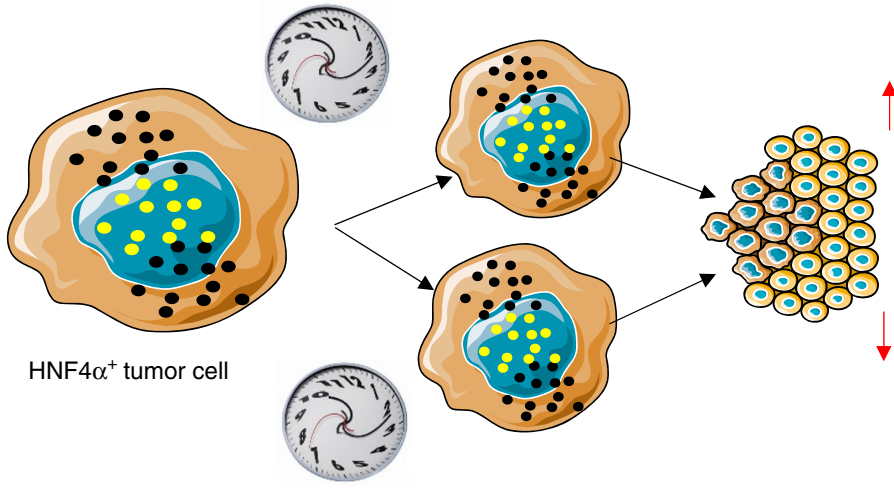

1. P2-HNF4 $\alpha$ expression

4 2. Repression of ARNTL

(BMAL1) by nuclear P2

3. Cytoplasmic expression of $\mathrm{P} 1-\mathrm{HNF} 4 \alpha$

1. Expression of BMAL1

2. Nuclear P1-HNF $4 \alpha$

3. Circadian restraint of

$M Y C$, cyclin genes

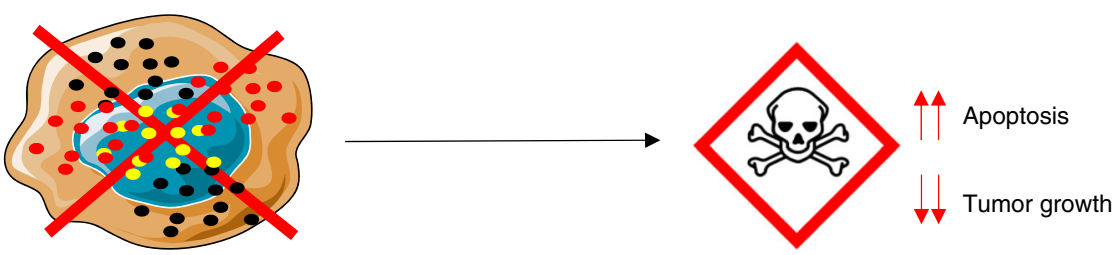

$\mathrm{HNF} 4 \alpha^{+}$tumor cell + BMAL1

Fig. 7 Model of HNF4 $\alpha$ isoform and BMAL1 expression in normal vs. cancer cells. In normal hepatocytes, the P1-HNF4 $\alpha$ isoform and the circadian protein BMAL1 are concomitantly expressed. While BMAL1 is found in both nuclear and cytoplasmic compartments, P1-HNF4 $\alpha$ is found exclusively in the nucleus, where it both activates and represses target genes, sometimes in a circadian manner. In HCC, P2-HNF4 $\alpha$ is induced, resulting in either the dual expression of both P1-HNF4 $\alpha$ and P2-HNF4 $\alpha$ or only P2-HNF4 $\alpha$ in HNF4 $\alpha$-positive tumors (approximately $50 \%$ of HCC). Induction of P2-HNF4 $\alpha$ results in direct transcriptional repression of BMAL1 and a large portion of P1-HNF4 $\alpha$ becomes cytoplasmic, reducing its ability to repress target cyclin and EMT genes in a circadian manner. Forced expression of BMAL1 in HNF4 $\alpha$-positive HCC results in a cell death and inhibition of tumor growth

F12 medium. Media was supplemented with $0.005 \mathrm{mg} \mathrm{ml}^{-1}$ insulin, $0.005 \mathrm{mg} \mathrm{ml}^{-1}$ transferrin, $5 \mathrm{ng} \mathrm{ml}^{-1}$ selenium, and $40 \mathrm{ng} \mathrm{ml}^{-1}$ dexamethasone. HEK

293 T cells were grown in Dulbecco's modified Eagle's medium, supplemented with $10 \%$ fetal bovine serum, 100 units $\mathrm{ml}^{-1}$ penicillin $\mathrm{G}$ sodium, and $100 \mu \mathrm{g} \mathrm{ml}^{-1}$ streptomycin.

For circadian synchronization by serum, cells were cultured in normal media until $90-100 \%$ confluency was reached. Cells were treated with media containing $50 \%$ horse serum for $2 \mathrm{~h}$. At the end of the serum shock treatment, cells were washed with PBS and the medium was replaced with normal assay medium. Application of $50 \%$ horse serum is considered ZT0; therefore, ZT0 refers to the unsynchronized condition.
Human HCC tissue arrays. Human tumor microarray slides containing lesions from patients with stages I-IV Hepatocellular carcinoma and matched nonmalignant tissues were obtained from UsBiomax.

Mouse HCC tissue slides. Paraffin slides containing liver sections from chronically jet-lagged mice with spontaneous hepatocellular carcinoma were obtained from Loning $\mathrm{Fu}^{17}$.

Mice. Care of animals was in strict accordance with guidelines from the McGovern Medical School, UTHealth Institutional Animal Care and Use Committee. Mice 
were group housed in standard pathogen-free conditions and fed ad libitum with a standard mouse chow (PicoLab Rodent Diet 5053) and water. Animals were maintained in steady 12 -h light/12-h dark cycles (24-h LD cycles). For experiments using livers from diet-induced obese mice, animals were fed with a diet containing $60 \%$ kcal from fat, Research Diets D12492, for 30 weeks starting at 8 weeks of age. $H n f 4 a^{F / F}$ and $S A^{+/ C r e-E R T 2}$ mouse lines were originally provided by Gonzalez ${ }^{25}$. To generate the conditional $H n f 4 a^{F / F ; A l b E R T 2 c r e}$ mice, $H n f 4 a^{F / F}$ mice were crossed with the tamoxifen-inducible hepatocyte-specific Cre recombinase expressing mouse $S A^{+/ C r e-E R T 2}$. Livers from 10-week-old male and female mice of each genotype were used for chromatin immunprecipitation.

Eight-week-old male NSG mice, NOD.Cg-Prkdcscid Il2rgtm1Wj1/SzJ were used for subcutaneous injections of HepG2 and SNU449 cells.

Mouse liver samples. Liver samples from a $7 \mathrm{HMZ}$ mice used in the study were provided by Frances Sladek. a7HMZ mice are described in (Briançon and Weiss, 2006). Three young adult males on standard lab chow, aged 16-20 weeks, were sacrificed at 10:30 (ZT 3.5), 13:30 (ZT 6.5) and 20:30 (ZT 13.5) (lights on at 7:00 and off at 19:00) for liver RNA-seq analysis.

Livers from C57BL/6J mice (The Jackson Laboratory, 000664) under normal chow (PicoLab Rodent Diet 5053) or made obese with HFD (Research Diets D12492) were obtained from mice at 10 months of age. Mice had been maintained on their respective diets from 2 months of age.

Plasmid constructs. The sources for all plasmid constructs are presented in Supplementary Table 2. Bmall, Hnf $4 \alpha 2$, and $H n f 4 \alpha 8$ were subcloned into pLenti CMV GFP Puro and pEGFP-C1. pLenti CMV GFP Puro was digested using BamHI and SalI. pEGFP-C1 was digested using XbaI and BamHI or Sall and XbaI followed by insertion of full length $H n f 4 \alpha 2$, Hnf $4 \alpha 8$,or Bmall amplified from their respective pcDNA vectors using primers designed with cohesive ends (Supplementary Table 9). Dephosphorylation of the linearized vector was performed using CIP (Alkaline Phosphatase, Calf Intestinal) followed by ligation of the vector with the corresponding fragment using a Quick Ligation kit for $15 \mathrm{~min}$ at room temperature according to manufacturer's protocol.

Transient transfection and siRNA, Transient transfection of plasmid vectors (Supplementary Table 2) was performed with Lipofectamine 2000 according to the manufacturer's protocol. Briefly, $1 \times 10^{6}$ cells were transfected with $1 \mu \mathrm{g}$ of plasmid using $5 \mu \mathrm{l}$ of Lipofectamine 2000. Knockdown of human and mouse P1/P2-HNF4a, $\mathrm{P} 1-\mathrm{HNF} 4 \alpha$ and P2-HNF4 $\alpha$ was performed using siRNA duplexes ${ }^{28}$ purchased from Eurofins Genomics (Supplementary Table 3 ). Approximately $1 \times 10^{6}$ cells were transfected with 25 pmol of siRNA and $5 \mu$ of Lipofectamine RNAiMAX Transfection reagent. Scrambled siRNA with similar GC content was used as a control. Transfections were performed following the manufacturer's protocol.

Lentivirus production and titration, and generation of stable cell lines. High titer lentiviral vector stock was produced in HEK 293T cells by Lipofectamine 2000 Transient transfection. Briefly, $10 \mu \mathrm{g}$ of transfer vectors, $5 \mu \mathrm{g}$ of each packaging vector, and $2.5 \mu \mathrm{g}$ of envelope vector (Supplementary Table 2 ) along with $50 \mu \mathrm{l}$ of Lipofectamine were used to transfect $1 \times 10^{7} \mathrm{HEK} 293 \mathrm{~T}$ cells. Supernatant harvests were performed every $16 \mathrm{~h}$ for a total of three harvests and then pooled. Supernatant was filtered through $0.45 \mu \mathrm{m}$ pore size filters and concentrated using one volume of Lenti-X concentrator reagent in three volumes of clarified supernatant according to the manufacturer's protocol. The titration of lentivirus was assessed using a qPCR based approach. Woodchuck Hepatitis Virus Posttranscriptional Regulatory Element and albumin genes were used as targets for a SYBR greenbased real-time qPCR method to determine virus titer $^{67}$ (Supplementary Table 4). For stable cell line production, cells were infected with concentrated lentiviral stock at $\sim 50$ multiplicity of infection in the presence of $8 \mathrm{mg} \mathrm{m}^{-1}$ polybrene for $8 \mathrm{~h}$ at $37^{\circ} \mathrm{C}$. A $2 \mu \mathrm{g} \mathrm{ml}^{-1}$ puromycin was then added to eliminate uninfected cells. Media was replaced every 3-4 days with fresh, puromycin-containing medium. Stable lines were used as 3D spheroid cultures and injected into the xenograft model.

Transwell invasion assays. Cells were seeded into the upper chamber of a Transwell insert in serum-free medium at a density of 50,000 cells/well. For invasion assays, inserts were precoated with $2 \mathrm{mg} \mathrm{ml}^{-1}$ Matrigel. Medium containing $20 \%$ FBS was placed in the lower chamber as a chemoattractant, and cells were incubated for $24 \mathrm{~h}$ in a $\mathrm{CO}_{2}$ incubator. Non-migrating cells were removed from the upper chamber by scraping. Remaining cells were fixed with methanol for 10 -min and stained with $0.1 \%$ crystal violet. Cells were imaged using a Zeiss inverted light microscope and 4 randomly chosen fields chosen on the lower side of the filter were used for counting.

3D spheroid culture. For 3D Matrigel-embedded cultures, human and mouse HCC cells were trypsinized and resuspended in complete medium. Twenty-five microliter cell suspension containing $5 \times 10^{3}$ cells was added to $25 \mu$ of Matrigel with a protein concentration ranging from 7 to $7.5 \mu \mathrm{g} \mu^{-1}$. Matrigel/cell suspension was gently mixed and deposited into 24 -well uncoated plates (Nalge Nunc International). To grow single organoids via the hanging drop method ${ }^{68}$, the lid from a $60 \mathrm{~mm}$ tissue culture plate was removed and $5 \mathrm{ml}$ of PBS was placed in the bottom of the dish to act as a hydration chamber. The lid was inverted and $10 \mu$ media containing $1 \times 10^{3}$ cells was deposited onto the bottom of the lid. The lid was returned to its upright position on top of the PBS-filled bottom chamber and incubated at $37^{\circ} \mathrm{C}$ overnight. This cell drop was carefully removed via pipet and mixed with $50 \%$ Matrigel prior to being deposited on the bottom of an uncoated 24-well plate. In both conditions, deposited Matrigel drops were incubated at $37^{\circ} \mathrm{C}$ for $30 \mathrm{~min}$. until solidified and then covered with $1 \mathrm{ml}$ complete medium and grown at $37^{\circ} \mathrm{C}$ under $5 \% \mathrm{CO}_{2}$ for 15 days. The media was changed every 2 days ${ }^{69}$ Organoids were harvested and washed $4 \times$ in ice cold PBS to remove all Matrigel after a 15 day growth period, and subjected to protein analysis.

MTT assay. Cell viability was assessed by MTT cell proliferation assay using a CellTiter $96^{\oplus}$ Non-Radioactive Cell Proliferation Assay (MTT) kit. Plates were processed according to the manufacturer's instructions. Absorbance was read at $570 \mathrm{~nm}$ using a Tecan infinite M1000 reader.

Luciferase reporter assay. To analyze Bmal1 promoter activity, $1 \mu \mathrm{g}$ of each construct was co-transfected into $1 \times 10^{6} \mathrm{Hepal-clc7}$ cells using $5 \mu \mathrm{l}$ of Lipofectamine 2000 according to the manufacturer's manual. Luminescence was measured $48 \mathrm{~h}$ posttransfection using a Luciferase assay system. Briefly, cells were washed in PBS and lysed by rapid shaking on a plate shaker at 200 RPM for $10 \mathrm{~min}$. at RT in Luciferase Lysis buffer (Supplementary Table 5). Thirty microliters of cell lysate was added to $70 \mu \mathrm{l}$ of Luciferase React Buffer containing luciferin (Supplementary Table 5), and luciferase activity was measured immediately on a plate reader. The pGL3-Basic plasmid (promoter-less) was used in each experiment to determine the basal levels of luciferase expression. Each construct was tested in three independent transfection experiments. A beta-galactosidase measurement was used to normalize experiments for transfection efficiency using Lac Z. Betagalactosidase activity was measured by adding $30 \mu \mathrm{l}$ lysate to $70 \mu \mathrm{l}$ of prepared $\mathrm{Z}$ buffer (Supplementary Table 5) followed by a $37^{\circ} \mathrm{C}$ incubation for 5 -min. Reactions were stopped with $50 \mu \mathrm{l}$ of $1 \mathrm{M} \mathrm{NaHCO}_{3}$. Absorbance was read at $450 \mathrm{~nm}$ using a Tecan infinite M1000 plate reader.

RNA extraction, reverse transcription, and quantitative PCR. Total RNA was isolated from cells and liver using TRIzol reagent according to the manufacturer's protocol. One microgram of total RNA was used for cDNA synthesis using an iScript cDNA synthesis kit. Advanced Universal SYBR Green Super mix from BioRad was used for $\mathrm{qPCR}$ amplification using a Bio-Rad C1000. PCR protocol settings were as follows: $95^{\circ} \mathrm{C}$ for $30 \mathrm{~s}, 95^{\circ} \mathrm{C}$ for $10 \mathrm{~s}, 62^{\circ} \mathrm{C}$ for $30 \mathrm{~s}$, and then 39 cycles at $65^{\circ} \mathrm{C}$ for $31 \mathrm{~s}$ and $65^{\circ} \mathrm{C}$ for $5 \mathrm{~s}$. Ribosomal subunit $18 \mathrm{~s}$ expression was used as control. The fold change in mRNA expression for each gene was calculated using 2 $-\Delta \Delta \mathrm{C}$. Primers used for amplification are presented in Supplementary Table 6.

Fractionation and immunoblotting. For whole cell lysates, liver tissue was homogenized in RIPA lysis buffer (Supplementary Table 7) for $15 \mathrm{~s}$, using a MagNA Lyser (Roche, IN, USA). Cultured cells were sonicated (Qsonica, Newton, USA) for $10 \mathrm{~s}$ at $30 \%$ amplitude in RIPA lysis buffer. Samples were spun at $10,000 \times g$ for $10 \mathrm{~min}$ to eliminate insoluble material. The total protein levels of the lysates were determined using the bicinchoninic acid method. Protein extracts were analyzed using $8 \%$ sodium dodecyl sulfate polyacrylamide gel electrophoresis followed by transfer to the nitrocellulose membrane before staining with primary antibodies (Supplementary Table 8). Secondary antibodies conjugated to horseradish peroxidase and enhanced chemiluminescence substrate, or alternatively, florescent conjugated secondary antibodies were used for detection (Supplementary Table 8). All full length blots and gels are supplied in the supplementary figures.

For tissue fractionations, liver tissue was homogenized for $15 \mathrm{~s}$ in BA (Supplementary Table 7) using a MagNA Lyser. Samples were spun at low speed for $10 \mathrm{~min}$ and supernatant was saved for cytoplasmic fractions. Pellets were resuspended in $1 \mathrm{ml}$ of BA and spun at low speed for 20 min. Pellets were washed $2 \times$ with low-salt buffer (LSB) (Supplementary Table 7) and the final pellet was dissolved in $150 \mu \mathrm{LSB}$ per $100 \mu \mathrm{l}$ pellet. The resulting homogenate was resuspended vigorously in $2 \times$ volume high-salt buffer (Supplementary Table 7), and nutated for $1 \mathrm{~h}$ at $4^{\circ} \mathrm{C}$. Samples were spun for $20 \mathrm{~min}$ at $10,000 \times g$ and the soluble nuclear material (supernatant) was snap frozen in liquid nitrogen and stored at $-80^{\circ} \mathrm{C}$. Remaining pellets were resuspended in RIPA lysis buffer and sonicated (Qsonica, Newton, USA) for $10 \mathrm{~s}$ at 30\% amplitude. Samples were spun at $10,000 \times g$ for $5 \mathrm{~min}$ to remove insoluble material and the resulting chromatin material was stored at $-80^{\circ} \mathrm{C}$.

Immunofluorescence. Cells and 3D spheroids were plated on glass cover slips in a six-well plate and fixed with $3.7 \%$ of methanol-free formaldehyde for $10 \mathrm{~min}$. followed by permeabilization with $0.1 \%$ Triton X-100 for $5 \mathrm{~min}$. Cells were blocked with $3 \%$ BSA dissolved in $1 \times$ PBS (pH 7.4) for $1 \mathrm{~h}$. Cells were incubated overnight at $4{ }^{\circ} \mathrm{C}$ with specific primary antibodies (Supplementary Table 8) in 3\% BSA. Human HCC microarray and mouse liver paraffin slides were baked for $15 \mathrm{~min}$ at $65^{\circ} \mathrm{C}$ and samples were immersed in xylene for $20 \mathrm{~min}$ and sequentially rehydrated in $100,90,80$, and $70 \%$ alcohol series and rinsed with PBS. For antigen retrieval, slides were steamed for $30 \mathrm{~min}$ in $10 \mathrm{mM}$ citrate buffer ( $\mathrm{pH}$ 6). After blocking with 
$10 \%$ goat serum and $3 \%$ BSA in PBS for $1 \mathrm{~h}$, slides were incubated with primary antibody overnight at $4{ }^{\circ} \mathrm{C}$ (Supplementary Table 8 ). For both IF and IHC, primary staining was followed by appropriate secondary fluorescent antibody (Supplementary Table 8) at 1:500 in a dark chamber for $1 \mathrm{~h}$ at room temperature. Nuclei were stained using DAPI. Images were captured and processed using a Zeiss Fluorescent microscope and Zen software.

CHIP assay. Chromatin immunoprecipitation was performed as described in ref. ${ }^{70}$ with some modifications. One hundred milligrams of frozen tissue or cells in culture from a $15 \mathrm{~cm}$ dish were washed in PBS containing protease inhibitors ( $1 \%$ NP-40, $1 \mathrm{~mm}$ PMSF, $1 \mathrm{mM} \mathrm{NaF}, 400 \mathrm{mM}$ NAM, and $3.3 \mathrm{mM}$ TSA). Tissue was minced in PBS with inhibitors followed by cross-linking with DSG ( $2 \mathrm{mM}$ final) in PBS containing $\mathrm{MgCl}\left(10 \mathrm{mM}\right.$ final) for $45 \mathrm{~min}$ at $25^{\circ} \mathrm{C}$. Formaldehyde (1\% final) was added for additional cross-linking at $25^{\circ} \mathrm{C}$ for $15 \mathrm{~min}$. Samples were spun at low speed and resuspended in PBS with protease inhibitors for homogenization. Following homogenization, samples were spun at low speed and resuspended in $2 \mathrm{ml}$ ChIP sonication buffer (1\% Triton X-100, $50 \mathrm{mM}$ Tris, $\mathrm{pH} 8.1,5 \mathrm{mM}$ EDTA, $0.1 \%$ deoxycholate, $150 \mathrm{mM} \mathrm{NaCl}$ ) containing protease inhibitors. Following sonication, samples were spun at $16,000 \times g$ in a microcentrifuge and supernatants were diluted 1:4 in ChIP dilution buffer (0.01\% SDS, 1.1\% Triton X-100, $1.2 \mathrm{mM}$ EDTA, $16.7 \mathrm{mM}$ Tris [pH 8.1], $167 \mathrm{mM} \mathrm{NaCl}$ ), and immunoprecipitated with HNF4a antibodies (Supplementary Table 4) or control IgG overnight. Protein DNA complexes were captured with blocked protein G-agarose beads and following elution, cross-linking was reversed by heating at $65^{\circ} \mathrm{C}$ overnight. Proteins were digested by $0.17 \mu \mathrm{g} \mu \mathrm{l}^{-1}$ proteinase $\mathrm{K}$ (New England Biolabs), and the DNA was extracted with phenol-chloroform, precipitated with ethanol, and dissolved in $100 \mu \mathrm{l}$ Tris-EDTA buffer (10 mm Tris-Cl [pH 8.0], $1 \mathrm{~mm}$ EDTA). ChIP samples

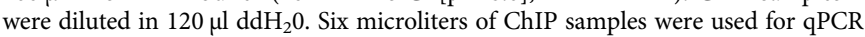
analysis using gene specific primers (Supplementary Table 9).

Xenograft experiments. HCC (SNU449) and hepatoblastoma cells (HepG2) stably expressing LUC were infected with pLenti-Gfp or pLenti-Gfp-Bmall. Fortyeight hours following infection, $2 \times 10^{6}$ cells were implanted subcutaneously into the right and left flanks.

Animals were monitored for luciferase expression $6 \mathrm{~h}$ following injection and then weekly for four consecutive weeks. For bioluminescent monitoring, $100 \mu \mathrm{l}$ of $50 \mathrm{mg} \mathrm{ml}^{-1} \mathrm{D}$-luciferin was injected intraperitoneally to the animals $15 \mathrm{~min}$ prior to luciferase measurements. Tumor growth was tracked by monitoring luciferase activity using an IVIS Spectrum (PerkinElmer, USA). Resulting tumors were excised at 4 weeks.

Tamoxifen injections. Hnf $4 a^{F / F ; ~ A l b E R T 2 c r e ~ m i c e ~ w e r e ~ i n j e c t e d ~ i n t r a p e r i t o n e a l l y ~}$ with tamoxifen $\left(10 \mathrm{mg} \mathrm{ml}^{-1}\right)$ in corn oil for 5 consecutive days (days 1-5) and then allowed to rest for 10 days prior to euthanization at the indicated zeitgeber times.

Quantification and statistical analysis. All results are expressed as the mean \pm SEM. Experiments with one variable were analyzed by unpaired Students $t$ tests or one-way ANOVA using Dunnetts multiple comparison test. Experiments involving two variables were analyzed by two-way ANOVA using Bonferroni post-tests (Prism 7.0). Significance was defined as a $P<0.05$. To test for rhythmicity, JTK_Cycle 48 was applied, using a window of $20-28$ h to capture circadian oscillations. For serum shock experiments, rhythmicity was ascertained from all values excluding the ZT0 (unsynchronized) time point

Fluorescent quantification. Fluorescent intensity of HNF4a or BMAL1 was measured using Fiji (Image J). Briefly, areas of interest were selected using the Drawing tools. From selected cells, the mean fluorescent densities were measured using "set measurement" in the analysis menu. Values were correcting using the total cell fluorescence (CTCF) values. CTCF $=$ mean fluorescent density of cells mean fluorescent density of background readings.

Expression profiling (RNA-seq) and analysis. RNA sequencing (RNA-seq) was carried out as previously described ${ }^{30}$. WT and $\alpha 7 \mathrm{HMZ}$ male mice were sacrificed $(N=3$, aged 16-18 weeks), at one of three time points, 10:30 AM, 1:30 PM, 8:30 PM (ZT 3.5, ZT 6.5, and ZT 13.5, respectively). The three mice were harvested in succession within a 30 min time frame. Livers were weighed and rinsed in PBS. Two $25 \mathrm{mg}$ pieces from each liver were immediately frozen in liquid nitrogen and stored at $-4{ }^{\circ} \mathrm{C}$. The miRNeasy Mini Kit (Qiagen) was used to extract and purify total RNA; $4 \mu \mathrm{g}$ of each sample was used to prepare a poly(A) + RNA library using TruSeq RNA Sample Prep v2 Kit (Illumina, Cat\# RS-122-2001). Libraries submitted for $75 \mathrm{bp}$ single-end sequencing with Illumina NextSeq 500 at the UCR IIGB Genomics Core. A total of 24 libraries ( 3 fed time points, 1 fasted time point, 2 genotypes each, and 3 replicates) were multiplexed and sequenced in two separate runs each of which yielded $\sim 600 \mathrm{M}$ reads, averaging $\sim 50 \mathrm{M}$ reads per sample.

Reads were aligned to the mouse reference genome, mm10, with Illumina's iGenome genes.gtf file using TopHat v2.1.1 with default parameters with the exception of allowing only one unique alignment for a given read, instead of the default 20. Raw read counts were calculated at the gene level for each sample using
HTSeq v0.6.1. Library normalization was performed with EDASeq. Within-lane normalization on GC content was performed with the loess method and between lane normalization was performed with the nonlinear full quantile method.

Normalization factors from EDASeq were used for differential expression analysis with DESeq2. Normalized read counts, FPKM (fragments per kilobase per million), and rlog (regularized log transformation) results were generated for downstream analysis. Pairwise contrasts for differential gene expression were generated for all relevant comparisons. Sample distance matrix were generated using rlog transformed values from DESeq2.

Detailed reagent information. Specific details related to all reagents and antibodies used can be found in Supplementary Table 10.

Image creation. Underlying cell images in Fig. 7 were taken from the Library of Science and Medical Illustraions, at http://www.somersault1824.com/resources/. A link to the license address is: https://creativecommons.org/licenses/by-nc-sa/4.0/.

\section{Data availability}

All data are publicly available. The genome-wide RNA-seq data has been deposited into Gene Expression Omnibus (GEO) and has a number of GSE117972. All relevant data are available from the authors associated with this manuscript and will be disseminated upon request.

Received: 29 January 2018 Accepted: 18 September 2018 Published online: 19 October 2018

\section{References}

1. Mazzanti, R., Arena, U. \& Tassi, R. Hepatocellular carcinoma: where are we? World J. Exp. Med. 6, 21-36 (2016).

2. Sun, B. \& Karin, M. Obesity, inflammation, and liver cancer. J. Hepatol. 56, 704-713 (2012).

3. Asher, G. \& Sassone-Corsi, P. Time for food: the intimate interplay between nutrition, metabolism, and the circadian clock. Cell 161, 84-92 (2015).

4. Bass, J. \& Takahashi, J. S. Circadian integration of metabolism and energetics. Science 330, 1349-1354 (2010).

5. Partch, C. L., Green, C. B. \& Takahashi, J. S. Molecular architecture of the mammalian circadian clock. Trends Cell Biol. 24, 90-99 (2014).

6. Takahashi, J. S. Transcriptional architecture of the mammalian circadian clock. Nat. Rev. Genet. 18, 164-179 (2017).

7. Roenneberg, T. \& Merrow, M. The circadian clock and human health. Curr. Biol. 26, R432-R443 (2016).

8. Filipski, E. et al. Host circadian clock as a control point in tumor progression. J. Natl Cancer Inst. 94, 690-697 (2002).

9. Filipski, E. et al. Circadian disruption accelerates liver carcinogenesis in mice Mutat. Res. 680, 95-105 (2009).

10. Mteyrek, A., Filipski, E., Guettier, C., Okyar, A. \& Levi, F. Clock gene Per2 as a controller of liver carcinogenesis. Oncotarget https://doi.org/10.18632/ oncotarget.11037 (2016).

11. Fu, L., Pelicano, H., Liu, J., Huang, P. \& Lee, C. The circadian gene Period 2 plays an important role in tumor suppression and DNA damage response in vivo. Cell 111, 41-50 (2002).

12. Winter, S. L., Bosnoyan-Collins, L., Pinnaduwage, D. \& Andrulis, I. L. Expression of the circadian clock genes Per1 and Per2 in sporadic and familial breast tumors. Neoplasia 9, 797-800 (2007).

13. Yang, X. et al. Downregulation of circadian clock gene Period 2 accelerates breast cancer growth by altering its daily growth rhythm. Breast Cancer Res Treat. 117, 423-431 (2009).

14. Altman, B. J. et al. MYC disrupts the circadian clock and metabolism in cancer cells. Cell Metab. 22, 1009-1019 (2015).

15. Jiang, W. et al. The circadian clock gene Bmall acts as a potential antioncogene in pancreatic cancer by activating the p53 tumor suppressor pathway. Cancer Lett. 371, 314-325 (2016).

16. Papagiannakopoulos, T. et al. Circadian rhythm disruption promotes lung tumorigenesis. Cell Metab. 24, 324-331 (2016).

17. Kettner, N. M. et al. Circadian homeostasis of liver metabolism suppresses hepatocarcinogenesis. Cancer Cell 30, 909-924 (2016).

18. Bunger, M. K. et al. Mop3 is an essential component of the master circadian pacemaker in mammals. Cell 103, 1009-1017 (2000).

19. Sladek, F. M., Zhong, W. M., Lai, E. \& Darnell, J. E. Jr. Liver-enriched transcription factor HNF-4 is a novel member of the steroid hormone receptor superfamily. Genes Dev. 4, 2353-2365 (1990).

20. Battle, M. A. et al. Hepatocyte nuclear factor 4 alpha orchestrates expression of cell adhesion proteins during the epithelial transformation of the developing liver. Proc. Natl Acad. Sci. USA 103, 8419-8424 (2006). 
21. Metzger, S., Halaas, J. L., Breslow, J. L. \& Sladek, F. M. Orphan receptor HNF4 and bZip protein C/EBP alpha bind to overlapping regions of the apolipoprotein B gene promoter and synergistically activate transcription. $J$. Biol. Chem. 268, 16831-16838 (1993).

22. Martinez-Jimenez, C. P., Kyrmizi, I., Cardot, P., Gonzalez, F. J. \& Talianidis, I. Hepatocyte nuclear factor 4 alpha coordinates a transcription factor network regulating hepatic fatty acid metabolism. Mol. Cell Biol. 30, 565-577 (2010).

23. Reddy, A. B. et al. Glucocorticoid signaling synchronizes the liver circadian transcriptome. Hepatology 45, 1478-1488 (2007).

24. Rhee, J. et al. Regulation of hepatic fasting response by PPARgamma coactivator-1alpha (PGC-1): requirement for hepatocyte nuclear factor 4 alpha in gluconeogenesis. Proc. Natl Acad. Sci. USA 100, 4012-4017 (2003).

25. Hayhurst, G. P., Lee, Y. H., Lambert, G., Ward, J. M. \& Gonzalez, F. J. Hepatocyte nuclear factor 4 alpha (nuclear receptor 2A1) is essential for maintenance of hepatic gene expression and lipid homeostasis. Mol. Cell Biol. 21, 1393-1403 (2001).

26. Barry, W. E. \& Thummel, C. S. The Drosophila HNF4 nuclear receptor promotes glucose-stimulated insulin secretion and mitochondrial function in adults. Elife https://doi.org/10.7554/eLife.11183 (2016).

27. Bonzo, J. A., Ferry, C. H., Matsubara, T., Kim, J. H. \& Gonzalez, F. J. Suppression of hepatocyte proliferation by hepatocyte nuclear factor 4 alpha in adult mice. J. Biol. Chem. 287, 7345-7356 (2012).

28. Chellappa, K. et al. Opposing roles of nuclear receptor HNF4alpha isoforms in colitis and colitis-associated colon cancer. eLife https://doi.org/10.7554/ eLife.10903 (2016).

29. Walesky, C. \& Apte, U. Role of hepatocyte nuclear factor 4 alpha (HNF4alpha) in cell proliferation and cancer. Gene Expr. 16, 101-108 (2015).

30. Vuong, L. M. et al. Differential effects of hepatocyte nuclear factor 4 alpha Isoforms on tumor growth and T-cell factor 4/AP-1 interactions in human colorectal cancer cells. Mol. Cell Biol. 35, 3471-3490 (2015).

31. Saandi, T. et al. Regulation of the tumor suppressor homeogene Cdx2 by HNF4alpha in intestinal cancer. Oncogene 32, 3782-3788 (2013).

32. Ning, B. F. et al. Hepatocyte nuclear factor 4 alpha suppresses the development of hepatocellular carcinoma. Cancer Res. 70, 7640-7651 (2010).

33. Lazarevich, N. L. et al. Progression of HCC in mice is associated with a downregulation in the expression of hepatocyte nuclear factors. Hepatology 39, 1038-1047 (2004).

34. Hatziapostolou, M. et al. An HNF4alpha-miRNA inflammatory feedback circuit regulates hepatocellular oncogenesis. Cell 147, 1233-1247 (2011).

35. Deane, N. G. et al. Hepatocellular carcinoma results from chronic cyclin D1 overexpression in transgenic mice. Cancer Res. 61, 5389-5395 (2001).

36. Lee, Y. K., Dell, H., Dowhan, D. H., Hadzopoulou-Cladaras, M. \& Moore, D. D. The orphan nuclear receptor SHP inhibits hepatocyte nuclear factor 4 and retinoid X receptor transactivation: two mechanisms for repression. Mol. Cell. Biol. 20, 187-195 (2000).

37. Ruse, M. D. Jr., Privalsky, M. L. \& Sladek, F. M. Competitive cofactor recruitment by orphan receptor hepatocyte nuclear factor 4alpha1: modulation by the F domain. Mol. Cell. Biol. 22, 1626-1638 (2002).

38. Walesky, C. et al. Hepatocyte nuclear factor 4 alpha deletion promotes diethylnitrosamine-induced hepatocellular carcinoma in rodents. Hepatology 57, 2480-2490 (2013).

39. Tanaka, T. et al. Dysregulated expression of $\mathrm{P} 1$ and $\mathrm{P} 2$ promoter-driven hepatocyte nuclear factor- 4 alpha in the pathogenesis of human cancer. $J$. Pathol. 208, 662-672 (2006).

40. Chellappa, K. et al. Src tyrosine kinase phosphorylation of nuclear receptor HNF4alpha correlates with isoform-specific loss of HNF4alpha in human colon cancer. Proc. Natl Acad. Sci. USA 109, 2302-2307 (2012).

41. Briancon, N. et al. Expression of the alpha7 isoform of hepatocyte nuclear factor (HNF) 4 is activated by HNF6/OC-2 and HNF1 and repressed by HNF4alphal in the liver. J. Biol. Chem. 279, 33398-33408 (2004).

42. Sun, K. et al. Phosphorylation of a conserved serine in the deoxyribonucleic acid binding domain of nuclear receptors alters intracellular localization. Mol. Endocrinol. 21, 1297-1311 (2007).

43. Soutoglou, E., Katrakili, N. \& Talianidis, I. Acetylation regulates transcription factor activity at multiple levels. Mol. Cell 5, 745-751 (2000).

44. Lopez-Terrada, D., Cheung, S. W., M.J., F. \& Knowles, B. B. Hep G2 is a hepatoblastoma-derived cell line. Hum. Pathol. 40, 1512-1515 (2009).

45. Pang, R. T. K. et al. Comparison of protein expression patterns between hepatocellular carcinoma cell lines and a hepatoblastoma cell line. Clin. Proteom. J. 1, 313-331 (2004).

46. Edmondson, R., Broglie, J. J., Adcock, A. F. \& Yang, L. Three-dimensional cell culture systems and their applications in drug discovery and cell-based biosensors. Assay Drug Dev. Technol. 12, 207-218 (2014).

47. Naugler, W. E. et al. Gender disparity in liver cancer due to sex differences in MyD88-dependent IL-6 production. Science 317, 121-124 (2007).
48. Hughes, M. E., Hogenesch, J. B. \& Kornacker, K. JTK_CYCLE: an efficient nonparametric algorithm for detecting rhythmic components in genome-scale data sets. J. Biol. Rhythms 25, 372-380 (2010).

49. Bae, S., Bessho, Y., Hojo, M. \& Kageyama, R. The bHLH gene Hes6, an inhibitor of Hes1, promotes neuronal differentiation. Development 127, 2933-2943 (2000).

50. Briancon, N. \& Weiss, M. C. In vivo role of the HNF4alpha AF-1 activation domain revealed by exon swapping. EMBO J. 25, 1253-1262 (2006).

51. Cai, S. H., Lu, S. X., Liu, L. L., Zhang, C. Z. \& Yun, J. P. Increased expression of hepatocyte nuclear factor 4 alpha transcribed by promoter 2 indicates a poor prognosis in hepatocellular carcinoma. Ther. Adv. Gastroenterol. 10, 761-771 (2017).

52. Cicchini, C. et al. Epigenetic control of EMT/MET dynamics: HNF4alpha impacts DNMT3s through miRs-29. Biochim. Biophys. Acta 1849, 919-929 (2015).

53. Zheng, N., Wei, W. \& Wang, Z. Emerging roles of FGF signaling in hepatocellular carcinoma. Transl. Cancer Res 5, 1-6 (2016).

54. Xiang, X. et al. Hepatocyte nuclear factor 4 alpha promotes the invasion, metastasis and angiogenesis of neuroblastoma cells via targeting matrix metalloproteinase 14. Cancer Lett. 359, 187-197 (2015).

55. Protiva, P. et al. Pigment epithelium-derived factor (PEDF) inhibits Wnt/betacatenin signaling in the liver. Cell Mol. Gastroenterol. Hepatol. 1, 535-549 (2015).

56. Matsumoto, K. et al. Antiangiogenic property of pigment epithelium-derived factor in hepatocellular carcinoma. Hepatology 40, 252-259 (2004).

57. Bu, Y. et al. A PERK-miR-211 axis suppresses circadian regulators and protein synthesis to promote cancer cell survival. Nat. Cell Biol. 20, 104-115 (2018).

58. Tang, Q. et al. Circadian clock gene Bmall inhibits tumorigenesis and increases paclitaxel sensitivity in tongue squamous cell carcinoma. Cancer Res. 77, 532-544 (2017).

59. Niu, L. et al. New insights into sorafenib resistance in hepatocellular carcinoma: Responsible mechanisms and promising strategies. Biochim. Biophys. Acta 1868, 564-570 (2017).

60. Armour, S. M. et al. An HDAC3-PROX1 corepressor module acts on HNF4alpha to control hepatic triglycerides. Nat. Commun. 8, 549 (2017).

61. He, B. et al. The small molecule nobiletin targets the molecular oscillator to enhance circadian rhythms and protect against metabolic syndrome. Cell Metab. 23, 610-621 (2016).

62. Hwang-Verslues, W. W. \& Sladek, F. M. HNF4alpha-role in drug metabolism and potential drug target? Curr. Opin. Pharmacol. 10, 698-705 (2010).

63. Yuan, X. et al. Identification of an endogenous ligand bound to a native orphan nuclear receptor. PLoS ONE 4, e5609 (2009).

64. Hertz, R., Magenheim, J., Berman, I. \& Bar-Tana, J. Fatty acyl-CoA thioesters are ligands of hepatic nuclear factor-4 alpha. Nature 392, 512-516 (1998).

65. Margini, C. \& Dufour, J. F. The story of HCC in NAFLD: from epidemiology, across pathogenesis, to prevention and treatment. Liver. Int. 36, 317-324 (2016).

66. Baffy, G., Brunt, E. M. \& Caldwell, S. H. Hepatocellular carcinoma in nonalcoholic fatty liver disease: an emerging menace. J. Hepatol. 56, 1384-1391 (2012).

67. Barczak, W., Suchorska, W., Rubis, B. \& Kulcenty, K. Universal real-time PCR-based assay for lentiviral titration. Mol. Biotechnol. 57, 195-200 (2015)

68. Foty, R. A simple hanging drop cell culture protocol for generation of 3D spheroids. J. Vis. Exp. https://doi.org/10.3791/2720 (2011).

69. Molina-Jimenez, F. et al. Matrigel-embedded 3D culture of Huh-7 cells as a hepatocyte-like polarized system to study hepatitis C virus cycle. Virology $\mathbf{4 2 5}$, 31-39 (2012).

70. Eckel-Mahan, K. L. et al. Reprogramming of the circadian clock by nutritional challenge. Cell 155, 1464-1478 (2013).

\section{Acknowledgments}

For technical help with reagents or mice, we would like to acknowledge Chris Janssen, Wenliang Li, Shin Yamazaki, Kavitha Rajendran, Sheng Zhang, Nadine Matthias, Naoki Nakayama, Ju-Seog Lee, and Radbod Darobi. We also thank Vihang Narkar for $d b / d b$ mouse livers and lentiviral packaging constructs. The following grants were used to support this study: NIDDK grants DK092590 and DK053895 (to R.B.), DK109001 (to K. S.), DK09470 and DK053895 (to F.M.S.), DK114037 (to K.E.-M.), and ACS grant RSG17-215-01-C (to K.E.-M.). Additional funds were provided by McGovern Medical School, part of UT Health Science Center at Houston (to K.E.M.) and the Harry E. Bovay Jr. Foundation (to M.G.K.).

\section{Author contributions}

Conceptualization: B.F. and K.E.-M.; Methodology: B.F., P.P., and K.E.-M.; Investigation: B. F., J.R.D., A.R.-L., C.K., and C.B.; Data analysis: B.F., S.-H.Y., J.R.D., F.M.S., M.G.K., R.B., K. E.-M., and S.H.W.; Writing-Original Draft: B.F. and K.E.-M.; Writing-Review and 
editing: K.S., F.M.S., M.G.K., R.B., and K.E.-M.; Funding acquisition: F.M.S. and K.E.-M.; Resources: S.-H.Y., R.B., F.M.S, L.F., and K.E.-M.; Supervision: F.M.S. and K.E.-M.

\section{Additional information}

Supplementary Information accompanies this paper at https://doi.org/10.1038/s41467018-06648-6.

Competing interests: The authors have the following interests to report: F.M.S. is currently or has previously consulted for Ambys, Cell Design Labs (now part of Gilead Science). The remaining authors declare no competing interests.

Reprints and permission information is available online at http://npg.nature.com/ reprintsandpermissions/

Publisher's note: Springer Nature remains neutral with regard to jurisdictional claims in published maps and institutional affiliations. (c) (i) Open Access This article is licensed under a Creative Commons Attribution 4.0 International License, which permits use, sharing, adaptation, distribution and reproduction in any medium or format, as long as you give appropriate credit to the original author(s) and the source, provide a link to the Creative Commons license, and indicate if changes were made. The images or other third party material in this article are included in the article's Creative Commons license, unless indicated otherwise in a credit line to the material. If material is not included in the article's Creative Commons license and your intended use is not permitted by statutory regulation or exceeds the permitted use, you will need to obtain permission directly from the copyright holder. To view a copy of this license, visit http://creativecommons.org/ licenses/by/4.0/.

(C) The Author(s) 2018 